\title{
Genome-wide identification and expression analysis of NADPH oxidase genes in response to ABA and abiotic stresses, and in fibre formation in Gossypium
}

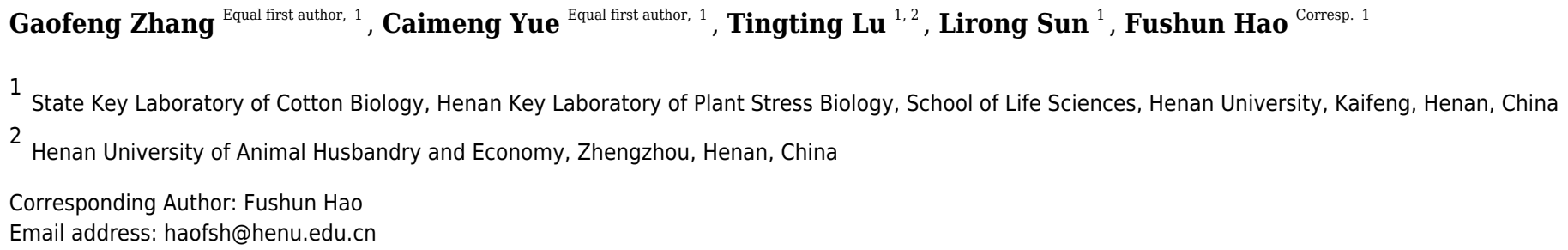

Plasma membrane NADPH oxidases, also named respiratory burst oxidase homologues (Rbohs), play pivotal roles in many aspects of growth and development, as well as in responses to hormone signalings and various biotic and abiotic stresses. Although Rbohs family members have been identified in several plants, little is known about Rbohs in Gossypium. In this report, we characterized 13, 13, 26 and 19 Rbohs in G. arboretum, G. raimondii, G. hirsutum and G. barbadense, respectively. These Rbohs were conservative in physical properties, structures of genes and motifs. The expansion and evolution of the Rbohs dominantly depended on segmental duplication, and were under the purifying selection. Transcription analyses showed that GhRbohs were expressed in various tissues, and most GhRbohs were highly expressed in flowers. Moreover, different GhRbohs had very diverse expression patterns in response to $A B A$, high salinity, osmotic stress and heat stress. Some GhRbohs were preferentially and specifically expressed during ovule growth and fiber formation. These results suggest that GhRbohs may serve highly differential roles in mediating ABA signaling, in acclimation to environmental stimuli, and in fiber growth and development. Our findings are valuable for further elucidating the functions and regulation mechanisms of the Rbohs in adaptation to diverse stresses, and in growth and development in Gossypium. 
1 Title page

2 Title:

3 Genome-wide identification and expression analysis of NADPH oxidase genes

4 in response to $\mathrm{ABA}$ and abiotic stresses, and in fibre formation in Gossypium

5 Authors:

6 Gaofeng Zhang ${ }^{1 \dagger}$, Caimeng Yue ${ }^{1 \dagger}$, Tingting Lu ${ }^{1,2}$, Lirong Sun ${ }^{1}$ and Fushun

$7 \mathrm{Hao}^{1 *}$

81 State Key Laboratory of Cotton Biology, Henan Key Laboratory of Plant

9 Stress Biology, School of Life Sciences, Henan University, Kaifeng 475004,

10 China

112 Henan University of Animal Husbandry and Economy, Zhengzhou 450046,

12 China

13 †These authors contributed equally to this work

14 Gaofeng Zhang: 2604035350@qq.com

15 Caimeng Yue: 2040499007@qq.com

16 Tingting Lu: 4147505@qq.com

17 Lirong Sun: 616805978@qq.com

*Corresponding author

Fushun Hao: haofsh@henu.edu.cn

Tel. 86-371-23881387; Fax 86-371-23881387 
Genome-wide identification and expression analysis of NADPH oxidase genes in response to $\mathrm{ABA}$ and abiotic stresses, and in fibre formation in Gossypium

Gaofeng Zhang ${ }^{1 \dagger}$, Caimeng Yue ${ }^{1 \dagger}$, Tingting Lu ${ }^{1,2}$, Lirong Sun ${ }^{1}$ and Fushun Hao ${ }^{1 *}$ College of Life Sciences, Henan University, Kaifeng 475004, China

2 Henan University of Animal Husbandry and Economy, Zhengzhou 450046, China

\section{ABSTRACT}

Plasma membrane NADPH oxidases, also named respiratory burst oxidase homologues (Rbohs), play pivotal roles in many aspects of growth and development, as well as in responses to hormone signalings and various biotic and abiotic stresses. Although Rbohs family members have been identified in several plants, little is known about Rbohs in Gossypium. In this report, we characterized 13, 13, 26 and 19 Rbohs in G. arboretum, G. raimondii, G. hirsutum and G. barbadense, respectively. These Rbohs were conservative in physical properties, structures of genes and motifs. The expansion and evolution of the Rbohs dominantly depended on segmental duplication, and were under the purifying selection. Transcription analyses showed that GhRbohs were expressed in various tissues, and most GhRbohs were highly expressed in flowers. Moreover, different GhRbohs had very diverse expression patterns in response to ABA, high salinity, osmotic stress and heat stress. Some GhRbohs were preferentially and specifically 
44 expressed during ovule growth and fiber formation. These results suggest that GhRbohs may

45 serve highly differential roles in mediating ABA signaling, in acclimation to environmental

46 stimuli, and in fiber growth and development. Our findings are valuable for further elucidating

47 the functions and regulation mechanisms of the Rbohs in adaptation to diverse stresses, and in

48 growth and development in Gossypium.

Keywords: NADPH oxidase, GhRboh, Gossypium, Reactive oxygen species, Gene family,

Phylogeny

\section{INTRODUCTION}

Reactive oxygen species (ROS) like superoxide radical $\left(\mathrm{O}_{2}{ }^{--}\right)$and hydrogen peroxide $\left(\mathrm{H}_{2} \mathrm{O}_{2}\right)$ are toxic byproducts of plant metabolisms. They also as signal molecules play pivotal roles in regulating plant growth and development, and in responding to hormone signals, and various biotic and abiotic stresses (Song et al., 2014; Mhamdi and Breusegem, 2018; Qi et al., 2018). oxidases (NOXs), also named respiratory burst oxidase homologues (Rbohs), have been demonstrated to be significant sources of ROS in plants under normal and stress conditions. The Rbohs transfers electrons from cytoplasmic $\mathrm{NAD}(\mathrm{P}) \mathrm{H}$ to $\mathrm{O}_{2}$ to generate $\mathrm{O}_{2}{ }^{\cdot-}$, followed by dismutation of the $\mathrm{O}_{2}{ }^{--}$to $\mathrm{H}_{2} \mathrm{O}_{2}$ (Suzuki et al., 2011; Marino et al., 2012; Chen and Yang, 2019). A large body of evidence indicates that Rbohs are vital regulators of many key cellular processes including vegetative and reproductive development, stomatal movement, and responses to 
hormones and diverse environmental stimuli in plants (Suzuki et al., 2011; Marino et al., 2012;

Xia et al., 2015; Chen and Yang, 2019; Sun et al., 2019). In Arabidopsis, 10 NOX homologs

(AtRbohA-AtRbohJ) have been detected. AtRbohB is implicated in modulating seed afterripening (Müller et al., 2009). AtRbohC mainly regulates root hair growth and cellular integrity (Foreman et al., 2003; Macpherson et al., 2008). Both AtrbohD and AtrbohF play key roles in root formation, stomatal closure, and in adaptation to multiple biotic and abiotic stresses (Ma et al., 2012; Marino et al., 2012; Jiao et al., 2013; Li et al., 2015; Liu et al., 2017; Sun et al., 2018; Chen and Yang, 2019; Wang et al, 2019). AtRbohE functions in tapetal programmed cell death and pollen development (Xie et al., 2014), and AtRbohI acts in drought stress response in seeds and roots (He et al., 2017). Both AtRbohH and AtRbohJ exert effects in pollen tube tip growth and polar root hair growth (Kaya et al., 2014; Mangano et al., 2017). Rbohs have also been identified and investigated in many other plants such as tobacco, potato, tomato, Medicago and maize. They regulate plant growth and development, and acclimations to different environmental stresses (Marino et al., 2012; Xia et al., 2015). In recent years, Rboh gene families have been characterized at genome-wide levels in many plants such as Arabidopsis, rice, wheat, Glycine max, Brassica rapa and multiple fruit trees. The expression patterns of the genes were also studied in tissues and in responding to diverse stresses (Chang et al., 2016; Hu et al., 2018; Cheng et al., 2019; Li et al., 2019; Liu et al., 2019; Navathe et al., 2019; Zou et al., 2019). Moreover, 2 RbohBs, 5 RbohDs (3 are RbohKs according to our classification) and 4 RbohFs in cotton have shown to be differentially expressed after infection by Verticillium dahliae (Li et al, 2019). However, knowledge about genomic information and genetic evolution of Rbohs in 
Gossypium is lacking, and their expression profiles in response to abiotic stresses in cotton remain to be explored.

Cotton is the most important fiber crop, and greatly contributes to the development of textile industry and national economy. Its growth and development as well as the yield and quality of fibers are significantly affected by various unfavorable environmental factors including drought, salinity and heat stress, and by ROS (Potikha et al., 1999; Allen., 2010). Therefore, it is great necessary to determine the roles and mechanisms of Gossypium Rbohs functioning in acclimation to stresses, and in fiber development.

Here, we conduct a genome-wide and comprehensive survey of the Rboh families in $G$. arboretum (A2), G. raimondii (D5), and their derived tetraploid species G. hirsutum (AD1) and G. barbadense (AD2). The expression patterns of Rboh genes were also analyzed in different tissues, in response to ABA, salinity, osmotic stress and heat stress, and in fiber formation in cotton. The research work will provide valuable information for further investigation of the roles of Rbohs in Gossypium.

\section{MATERIALS AND METHODS}

\section{Identification of Gossypium Rboh family members}

The amino acid sequences of 10 Arabidopsis Rbohs (AtRbohA-J) were used as queries to search against the genome sequence databases of $G$. arboretum (BGI-CGB v2.0 assembly genome), $G$. raimondii (JGI assembly v2.0 data.), G. hirsutum (NAU-NBI v1.1 assembly genome) (www.cottongen.org), and G. barbadense (http://database.chgc.sh.cn/cotton/index.html), respectively, applying the BLAST program with default setting $\left(E-v a l u e<\mathrm{e}^{-10}\right)($ Camacho et al., 
107 2009). The Gossypium Rbohs were confirmed again in NCBI

108 (https:/www.ncbi.nlm.nih.gov/Structure/cdd/wrpsb.cgi) and the SMART programme 109 (http://smart.embl-heidelberg.de/) by analyzing the conserved domains. The questionable Rbohs 110 annotations were manually reassessed.

111 The properties of the Gossypium Rbohs were predicted by the online tool ExPaSy 112 (http://web.expasy.org/protparam/). The structures of the Rbohs were identified by the GSDS 113 software (http:/gsds.cbi.pku.edu.cn). The conserved domains of Gossypium Rbohs were 114 determined using the Pfam (http://pfam.xfam.org/) and NCBI web CD-search tool 115 (http://www.ncbi.nlm.nih.gov/Structure/bwrpsb/bwrpsb.cgi). The Adobe Illustrator CC software 116 was used to depict the structures of genes and conserved domains of proteins.

117 The chromosomal distributions of the Rbohs were characterized on the basis of genome 118 annotation described above. The MapInspect software 119 (http://www.mybiosoftware.com/mapinspect-compare-display-linkage-maps.html) was used to 120 visualize the locations of the Rbohs.

121 Phylogenetic analysis of Rbohs

122 The genome sequences and gene annotation databases of Rbohs were retrieved from the websites 123 for Arabidopsis thaliana (http://www.arabidopsis.org), Theobroma cacao

124 (http://cocoagendb.cirad.fr), Ricinus communis (http://castorbean.jcvi.org), Populus trichocarpa 125 (http://www.phytozome.net/poplar), Glycine max

126 (https://phytozome.jgi.doe.gov/pz/portal.html\#!info?alias=Org_Gmax), Brachypodium 127 distachyon (http://plants.ensembl.org/Brachypodium_distachyon/Info/Index), Oryza sativa 
128 (http://rapdb.dna.affrc.go.jp), and the four Gossypium plants. The amino acid sequences of

129 Rbohs were aligned by the MUSCLE software (https:/www.ebi.ac.uk/Tools/msa/muscle/). The

130 phylogenetic trees were made applying the maximum-likelihood (ML) method and the IQ-TREE

131 server, in which the ModelFinder method was used to select the best model. Models JTT+G4 and

$132 \mathrm{JTT}+\mathrm{R} 7$ were respectively utilized to generate the unrooted phylogenetic tree for the Gossypium

133 Rbohs and the multiple species phylogenetic tree of Rbohs. The ultrafast bootstrap

134 approximation was also used (Minh et al., 2013; Nguyen et al., 2015; Kalyaanamoorthy et al.,

135 2017; Lu et al., 2019).

136 Synteny and $K a / K s$ analysis

137 The values of nucleotide substitution parameter $K a$ (non-synonymous) and $K s$ (synonymous) for

138 Gossypium Rbohs were calculated with the PAML program

139 (http://abacus.gene.ucl.ac.uk/software/paml.html). The homologous genes were searched by the

140 MCScanx software (http://chibba.pgml.uga.edu/mcscan2). The syntenic maps of the Rbohs from

141 G. arboretum, G. raimondii and G. hirsutum were generated using the circos- $0.69 \pm 3$ software

142 package with default parameters (http://www.circos.ca).

143 Expression analysis of Rbohs in tissues and in responding to ABA, salinity, osmotic stress

144 or heat stress in cotton

145 To examine the transcriptional abundances of Rbohs in different tissues, samples of roots, stems

146 and leaves were collected from TM-1 cotton plants grown in nutrient soil (rich soil:vermiculite =

$1472: 1, \mathrm{v} / \mathrm{v}$ ) for $21 \mathrm{~d}$ in a growth chamber. Flowers were isolated in the morning at the first day of

148 anthesis. The fibers were obtained from the ovules at about 23 dpa (day post anthesis). To

Peer) reviewing PDF | (2019:08:39951:2:0:NEW 8 Dec 2019) 
149

150

151

152

153

154

155

156

157

158

159

160

161

162

163

164

165

166

167

168

169

monitor the expression of Rbohs in responding to $\mathrm{ABA}$ or abiotic stresses, the cotton seedlings were cultivated in liquid 1/2 MS medium for three weeks (Lu et al., 2017). The seedlings were then treated with $100 \mu \mathrm{M} \mathrm{ABA}, 200 \mathrm{mM} \mathrm{NaCl}, 10 \%$ PEG6000 or high temperature $\left(42^{\circ} \mathrm{C}\right)$ for 3 , 6,12 and $24 \mathrm{~h}$, respectively. The plants without treatment by ABA or a stress were used as controls. The cotton roots for $\mathrm{ABA}, \mathrm{NaCl}$ or PEG treatment, and leaves for heat stress were collected, immediately frozen in liquid nitrogen and stored at $-70^{\circ} \mathrm{C}$. Total RNA extraction, cDNA synthesis and quantitative real-time RT-PCR (qRT-PCR) analysis were performed as described previously (Lu et al., 2017; Zhang et al., 2017). The specific primers of cotton Rbohs for the qRT-PCR experiments were list in Table S1.

\section{The expression profiles of Rbohs in ovule and fiber development of G. hirsutum}

The GhRbohs transcriptomic data during ovule growth and fiber formation were obtained from the website http://structuralbiology.cau.edu.cn/gossypium/, and the heatmaps were generated using TBtools v0.6672 (Chen et al., 2018).

\section{RESULTS}

\section{Genome-wide survey of Rbohs in four Gossypium species}

Based on the available database and bioinformatic analysis results, a total of 13, 13, 26 and 19

Rboh members were identified in G. arboretum, G. raimondii, G. hirsutum and G. barbadense, respectively (Table 1). The Rbohs in G. arboretum and G. raimondii were respectively nominated according to their orthologous similarity in amino acid sequences to the 10 Arabidopsis AtRbohs whereas Rbohs in the two tetraploid Gossypium genres were named based on their homologous similarity in amino acid sequences to the Rbohs of the two diploid species 
170 following the methods of Mohanta et al., (2015) and Zhang et al., (2017) in other genes. That is,

171 to name a Gossypium Rboh gene, we used the first letter of the genus (upper case) and that of the

172 species (lower case), followed by RbohA-J, RbohK or RbohL. The RbohA-J was the same as the

173 name of its ortholog in Arabidopsis. RbohK and RbohL were new paralogous members different

174 from Arabidopsis orthologs. If there were several Gossypium Rboh orthologs for an AtRboh or

175 other Rboh, a hyphen and different numbers (1, 2, 3 or 4) were added to difine the different

176 paralogs. For a Rboh of G. hirsutum or G. barbadense, the letter A or D was added at the end of

177 the name to indicate the gene in A or D genome (Table 1).

178 The Gossypium Rbohs showed conservative physical properties (Table 1). Most of the Rbohs

179 are similar in amino acid (AA) lengths, molecular weights (MWs), and theoretical isoelectric

180 points (pI). There were no significant differences in these parameters among the four species.

181 The GaRbohs, GrRbohs, GhRbohs and GbRbohs had 803-933, 786-943, 721-940, and 833-985

182 AA, respectively, with the exception of GaRbohE (1015 AA), GaRbohH (1273 AA), and

183 GbRboh-4D (688 AA). The MWs of the Rbohs varied from $78.3 \mathrm{kDa}$ to $145 \mathrm{kDa}$ with an

184 average of 101.3. The pIs of the Rbohs ranged from 8.65 to 9.63 (Table 1).

\section{Phylogeny and structure of Gossypium Rbohs}

We constructed an unrooted phylogenetic tree for the 71 Gossypium Rbohs, and analyzed their

evolutionary relationship between G. arboreum, G. raimondii and G. hirsutum, G. barbadense.

As shown in Fig.1A, the Rbohs can be categorized into seven subfamilies (I to VII). Each of the

subfamily I, III, and VI contained 6 members. They were respective homologs of AtRbohE,

AtRbohB and AtRbohH in the four Gossypium species. Both II and IV had 15 members, which

Peer] reviewing PDF | (2019:08:39951:2:0:NEW 8 Dec 2019) 
191

192

193

194

195

196

197

198

199

200

201

202

203

204

205

206

207

208

209

210

211

respectively belonged to the orthologs of AtRbohF and AtRbohD in Gossypium. Subfamily V

and VII were composed of 18 and 5 Rbohs, respectively. They were homologous members of

AtRbohC and AtRbohJ of Gossypium, respectively. Unexpectedly, no orthologs of AtRbohA,

AtRbohG and AtRbohI were detected in the tested Gossipium plants. We found that all the

homologs of an AtRboh in each of the four Gossypium species commonly grouped together,

reflecting their closer genetic and evolutionary relationship. Moreover, the majority of GaRbohs

and GrRbohs had their corresponding homologs in G. hirsutum and G. barbadense. However, no

orthologs of GaRbohD-3 and GrRbohD-3 were detected in G. barbadense.

The exon/intron structures of the Gossypium Rbohs were analyzed to define the possible

evolutionary mechanisms of the genes. It was found that 21, 22 and 15 Rbohs individually had

12, 14 and 11 exons. Other Rboh genes harbored 13 (for 6 Rbohs), 15 (for 4 Rbohs) or 10 (for 3

Rbohs) exons, respectively. Generally, the introns of the Rbohs were short in length. However,

GhRbohF-2A possesses a very long intron. Additionally, the Gossypium Rbohs in the same

subfamily frequently had similar number of exons, and similar exon/intron organizations. For

example, all Rboh members of subfamily III contain 12 exons, and each Rbohs from subfamily

VII carried 14 exons. The exon/intron structures for many members of one subfamily were alike

(Fig. 1B). Regarding the number and organization of exon/intron, about half of GhRbohs were

very similar to or the same as their orthologs in G. arboreum and G. raimondii. However, few of

GbRbohs were very similar to their orthologs in G. arboreum and G. raimondii, suggesting more

distant evolutionary relationship between GbRbohs and GaRbohs or GrRbohs.

To determine the motif compositions, we studied the putative domains in the Gossypium

Peer) reviewing PDF | (2019:08:39951:2:0:NEW 8 Dec 2019) 
212 Rbohs. Sixty out of the 71 Rbohs had the four characteristic motifs of plant Rboh family

213 (NADPH_Ox, Ferric_reduct, FAD_binding_8 and NAD_binding_6), and most of the Rbohs

214 contained the calcium-binding EF-hand motifs. However, the ferric_reduct motif was absent in

215 GhRbohK-3A, GrRbohL, GhRbohLD and GbRbohLD. Also, the FAD_binding_8 motif did not

216 exist in GhRbohED, GhRbohEA, GhRbohF-2A, GaRbohF-2 and GbRbohHD, and the

217 NAD_binding_6 motif did not exist in GhRbohD-1A (Fig. 1C).

218 Chromosomal distributions of Rbohs

219 We analyzed the locations of Rbohs in chromosomes from the Gossypium plants, and found that

220 the Rbohs were unevenly distributed on different chromosomes (Fig. 2). Among these, 10

221 GaRbohs, 13 GrRbohs, 22 GhRbohs and 19 GbRbohs were separately located on 7, 7, 14 and 13

222 chromosomes, respectively. Three genes were present in each of the D01, D08, At07, Dt07,

223 At'07 and Dt'07 chromosomes. Each of 11 chromosomes (A06, A07, A10, D03, D09, At05,

224 Dt03, Dt05, Dt12, At'12 and Dt'12) harbored 2 genes (Fig. 2). Other chromosomes individually

225 contained one gene. Additionally, the distributions of the Rbohs on individual chromosome were

226 irregular. Some genes were situated on the lower end of the chromosome arms, some on the

227 upper end or in the middle region (Fig. 2). Besides, 3 GaRbohs and 4 GhRbohs were located in

228 scaffolds.

229 We compared the distributions of GaRbohs and GrRbohs with their corresponding

230 homologous genes in G. hirsutum and G. barbadense in chromosomes. Unexpectedly, the

231 majority of the GhRbohs and GbRbohs did not distribute their corresponding homologous

232 chromosomes in G. arboreum and G. raimondii. Only the positions of GhRbohK-3D, GhRbohF- 
$2331 D$ and GbRbohK-3D in chromosomes were matched with those of GrRbohK-3, GrRbohF-1 and

234 GrRbohK-3, respectively (Fig. 2). Our findings suggest that many complex conversion events

235 occur in homoeologous chromosomes harboring Rbohs among the Gossypium species during the

236 long-term evolution.

\section{Synteny analysis of $R$ bohs}

238 Gene duplications including tandem and segmental duplications are essential for the expansion

239 of gene family during evolution (Cannon et al., 2004). In order to understand the genetic

240 evolutionary relationship among the Gossypium Rbohs, we characterized the homologous gene

241 pairs of Rbohs from G. arboreum, G. raimondii and G. hirsutum, and analyzed the collinear

242 relationships. A total of 152 homologous pairs were detected among the three species. Twenty-

243 four gene pairs were present between G. arboretum and G. hirsutum, 45 between G. raimondii

244 and $G$. hirsutum, and 25 between $G$. arboretum and G. raimondii, 19 between the At-genome

245 and Dt-genome of $G$. hirsutum, and a few within all the G. arboretum, G. raimondii and $G$.

246 hirsutum species (Fig. 3). These homologous pairs belonged to 149 collinearity blocks. Most of

247 the blocks owned only one gene pair. However, homologous gene pairs GrRbohK-1/GhRbohK-

$2481 D$, GrRbohE/GhRbohED and GrRbohH/GhRbohHD between chromosome D01 and Dt07 were

249 in one block, and gene pairs GrRbohK-1/GhRbohK-1A and GrRbohH/GhRbohHA between

250 chromosome D01 and At07 were in a block. Also, gene pairs GrRbohK-2/GhRbohK-2D and

251 GrRbohL/GhRbohLD between chromosome D08 and Dt12 belonged to one block, and gene pairs

252 GhRbohK-1A/GhRbohK-1D and GhRbohHA/GhRbohHD between chromosome At07 and Dt07

253 belonged to one block. No one gene pair was involved in tandem duplication. These results

Peer) reviewing PDF | (2019:08:39951:2:0:NEW 8 Dec 2019) 
254 255 256 258 259 260 261

imply that segmental duplications play predominant roles in the formation of Gossypium Rbohs during evolution.

\section{Analysis of the $K a / K s$ values of Rbohs in G. arboreum, G. raimondii and G. hirsutum}

To gain insight into the divergence and selection in duplication of Rbohs after polyploidization, the non-synonymous $(\mathrm{Ka})$, synonymous $(\mathrm{Ks})$ and $K \mathrm{a} / K$ s values were calculated for the homologous gene pairs among G. arboreum, G. raimondii and G. hirsutum. As shown in Fig. 4, the average $K a / K s$ values among homologous gene pairs of the Rbohs between genomes and/or subgenomes AAt, DDt, ADt, DAt, AtDt and AD were 0.45, 0.31, 0.22, 0.30, 0.28 and 0.24, respectively, and that of all the gene pairs was 0.30 , clearly less than 1 (Fig. 4). These data indicate that all the Rbohs are mainly under the purifying selection during evolution.

\section{Phylogenetic relationship of Rbohs in Gossypium and other plants}

To examine the phylogenetic relationships of Rbohs among the four Gossypium species and other plants including A. thaliana, T. cacao, R. communis, P. trichocarpa, G. max, B. distachyon, and O. sativa, a phylogenetic tree for Rbohs of these plants was made (Fig. 5). Not surprisingly, most of the Gossypium Rbohs were clustered closely in a subfamily. Moreover, the Rbohs from Gossypium generally clustered closer with those from eudicots like A. thaliana and G. max than with those from monocots like $B$. distachyon, and $O$. sativa. The Rbohs from multiple dicotyledon species and those from $B$. distachyon and $O$. sativa were also frequently grouped in a branch (Fig. 5). Notably, the Rbohs from all the Gossypium species often clustered closely with those from T. cacao (Fig. 5), reflecting the closer evolutionary relationship of Gossypium Rbohs with cacao Rbohs. 


\section{Expression profiles of GhRbohs in tissues}

276 To better understand the potential roles of GhRbohs in different tissues, the expression patterns

277 of the 26 GhRbohs genes were studied using qRT-PCR. The results revealed that all genes were

preferentially expressed in flowers (Fig. 6A-X) except that GhRbohBD and GhRbohK-1D were

highly expressed in roots (Fig. 6Y-Z). The transcriptional levels of GhRbohBA, GhRbohD-1D

and GhRbohD-2D were high in roots whereas those of $G h R b o h K-3 D$ were high in leaves. The

transcripts of most genes were not abundant in stems and fibers (Fig. 6, Table S2). These data

indicate that the GhRbohs might exert key effects in flowers rather than in stems and fibers of

cotton.

\section{Expression patterns of GhRbohs in responses to ABA, high salinity, osmotic or heat stress}

The transcription changes of the GhRbohs were examined after treatments with $100 \mu \mathrm{M}$ ABA,

$200 \mathrm{mM} \mathrm{NaCl}, 10 \%$ PEG6000 or high temperature $\left(42^{\circ} \mathrm{C}\right)$ for different periods of time. In the

presence of exogenous ABA, the expression patterns of GhRbohs were diverse. The

transcriptional levels of 6 genes diminished at early time, but heightened at $12 \mathrm{~h}$ and/or $24 \mathrm{~h}$ (Fig.

7A-F). Ten GhRbohs showed increased transcription at $3 \mathrm{~h}$ or $6 \mathrm{~h}$, and decreased transcription

afterwards (Fig. 7G-P). Expression of 7 genes had decreasing trends in response to ABA (Fig.

7Q-W). By contrast, the expression of 3 genes (GhRbohK-2A, GhRbohF-1A and GhRbohLA)

displayed increasing trends in responding to ABA (Fig. 7X-Z, Table S3). 
296 showed diminished trends (Fig. 8Q-R). In contrast, the abundances of 8 GhRbohs exhibited

297 markedly increased trends over treatment time (Fig. 8S-Z, Table S4).

298 After treatment of cotton seedlings with PEG, the transcript abundances of 10 GhRbohs

299

300

301

302

303

304

305

306

307

308

309

310

311

312

313

314

315

316

markedly lowered at $3 \mathrm{~h}$ and $6 \mathrm{~h}$, and observably elevated at $12 \mathrm{~h}$ and/or $24 \mathrm{~h}$ (Fig. 9A-J). The

expression of 7 GhRbohs was upregulated at $3 \mathrm{~h}$ and/or $6 \mathrm{~h}$, then downregulated over time (Fig.

9K-Q). The transcriptional levels of 4 GhRbohs (GhRbohK-2A, GhRbohD-1D, GhRbohD-2A and

GhRbohED) showed dropped trends while those of the remaining 5 GhRbohs (GhRbohK-1D,

GhRbohK-3A, GhRbohK-3D, GhRbohD-3A and GhRbohD-3D) displayed increased trends over

time (Fig. 9R-U, V-Z) (Table S5).

After exposure of cotton seedlings to high temperature, the expression levels of 5 GhRbohs cut down over time till $12 \mathrm{~h}$, but increased at $24 \mathrm{~h}$ (Fig. 10A-E). Eighteen out of twenty-six GhRboh genes were upregulated at $3 \mathrm{~h}$ and/or $6 \mathrm{~h}$, but downregulated at $12 \mathrm{~h}$. At $24 \mathrm{~h}$, their transcripts were significantly abundant or few (Fig. 10F-W). Heat stress caused clear decreases in transcription levels of GhRbohK-1A and GhRbohK-1D but enhancements in expression levels of GhRbohF-2A (Fig. 10X-Y, Z) (Table S6).

Collectively, these results strongly suggest that GhRbohs play differential roles in sensing and transducing ABA signal and in diverse responses to salt stress, osmotic stress and high temperature, highlighting the great importance of NOXs in cotton tolerance to various abiotic stresses.

\section{Expression patterns of GhRbohs in the development of ovules and fibers}

To acertain whether GhRbohs play important roles in fiber development, the related 
317 transcriptome data were downloaded from the website

318 (http://structuralbiology.cau.edu.cn/gossypium/), and the expression profiles of all the 26

319 GhRbohs during the development of ovules and fibers were investigated. The results showed that

320 GhRbohK-1A, GhRbohK-1D, GhRbohK-2A, GhRbohK-2D, GhRbohK-3D, GhRbohD-3A,

$321 G h R b o h D-3 D$ and GhRbohF-3D were highly expressed in the early stages of ovule development,

322 and GhRbohK-2A, GhRbohK-2D, GhRbohD-3A, GhRbohD-3D and GhRbohF-3D were strongly

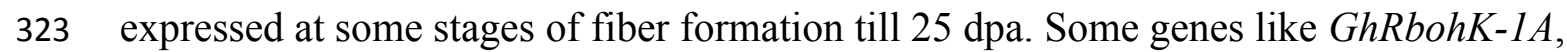

324 GhRbohK-1D, GhRbohK-3D, GhRbohF-1A, GhRbohF-1D, GhRbohF-3A, GhRbohLA and

$325 G h R b o h L D$ were expressed at some stages in the development of ovules and/or fibers. Other

326 GhRbohs were less expressed or not expressed during growth of ovules and fiber formation (Fig.

327 11).

\section{DISCUSSION}

329 In the present study, we identified 13, 13, 26 and 19 Rbohs in G. arboretum, G. raimondii, G.

hirsutum and G. barbadense, respectively (Table 1). The number of these Rbohs in Gossypium,

particularly in G. hirsutum and G. barbadense was larger than that in other plants such as

Arabidopsis (10), rice (9), maize (14), Brachypodium distachyon (9), Populus trichocarpa (10),

wheat (19), Brassica rapa (14) and soybean (17) (Chang et al., 2016, Hu et al., 2018; Li et al.,

2019; Liu et al., 2019; Navathe et al., 2019), implying that more sophisticated ROS signal

transduction mechanisms may exist in Gossypium. Compared with other crops as mentioned

above, Gossypium plants are more tolerant to environmental stresses like drought, high salt, heat 
338

required for the high tolerance of cotton to stresses.

We observed that all the GaRbohs and GrRbohs had their corresponding homologues in G.

hirsutum, and vice versa, highlighting the great importance of the GaRbohs and GrRbohs for survival of $G$. hirsutum after the polyploidization event. In contrast, GaRbohD-3 and GrRbohD-3

had no orthologs in G. barbadense, indicating that the two genes might not essential for growth and development of $G$. barbadense plants under relaxed selection pressures, and lost during evolution.

The homologous members of each AtRboh in the four Gossypium species always clustered together, and the exon/intron arrangements of GaRbohs and GrRbohs were very similar to those of the GhRbohs and GbRbohs within one subfamily. These suggest that the Gossypium Rbohs are originated from a common ancester. The number of exons for Gossypium Rbohs was 10-15, similar to that in Rbohs in Arabidopsis, rice, maize, wheat, canola and soybean (Chang et al., 2016; Hu et al., 2018; Li et al., 2019; Liu et al., 2019; Navathe et al., 2019). Additionally, the majority of the Gossypium Rbohs had the conserved EF hand domains, and NADPH_Ox, Ferric_reduct, FAD_binding_8 and NAD_binding_6 motifs of higher plants (Fig. 2). Our findings imply that Rbohs in plants are quite conservative during evolution.

We noticed that each of AtRbohD and AtRbohF had more homologues in the four Gossypium species. This coincided with the complex and more diverse roles of AtRbohD and AtRbohF in Arabidopsis. Similarly, no orthologs of AtRbohA, AtRbohG, AtRbohI, and AtRbohJ were found in Gossypium, in agreement with the small roles of these subunits in Arabidopsis. These data indicate that Gossypium RbohD and RbohF most likely have more important roles than other 
359

360

361

362

363

364

365

366

367

368

369

370

371

372

373

374

375

376

377

378

379

members in growth and development, and in adaptation to stresses.

Identification of gene distributions on chromosomes revealed that most GaRbohs and

GrRbohs did not match with their orthologs of G. hirsutum and G. barbadense in situated

chromosomes and in specific positions, hinting that very complex exchange events of

chromosome segments occur in the two tetraploid species after DNA polyploidization.

Synteny analysis showed that most of the GaRbohs and GrRbohs had many homologous gene

pairs in their homologs of the two tetraploid cotton plants, indicating that Gossypium Rboh

family members amplify mainly through segmental duplication during evolution. To further

examine the divergence and selection in duplication of $R b o h$ genes, the $K a / K s$ values were

analyzed. The results revealed that the average $K a / K s$ value for all the homologous gene pairs

among G. arboreum, G. raimondii and G. hirsutum was 0.3 , much small than 1 , suggesting that

these genes were majorly under the purifying selection during evolution.

We compared the homologous relationships of Rboh gene family among four cotton species, $A$.

thaliana, T. cacao, R. communis, P. trichocarpa, G. max, B. distachyon, and O. sativa using a

phylogenetic tree (Fig. 5), and found that the Rbohs from the dicotyledon and monocotyledon

commonly gather in one branch, hinting that these Rbohs emerge before the divergence of

eudicots and monocots. Moreover, dicot Rbohs always clustered together, and monocot Rbohs

also grouped together, indicating that dramatic alterations of Rboh genes occur after the isolation

of monocotyledons and dicotyledons. We also observed that Gossypium Rbohs clustered closer

with Rbohs from cocoa than with those from other plants, reflecting the closer evolutionary

relationship of Gossypium with cocoa. This means that the majority of homologous Rbohs from

PeerJ reviewing PDF | (2019:08:39951:2:0:NEW 8 Dec 2019) 
380

381

382

383

384

385

386

387

388

389

390

391

392

393

394

395

396

397

398

399

400

Gossypium and cacao may emerge before the separation of the two genera from one common

ancestor.

Transcription analysis showed that most GhRbohs were expressed in various tissues including roots, stems, leaves, flowers and fibers. Interestingly, the majority of genes were preferentially

expressed in the flower (Fig. 6, Table S2), quite different from the results obtained from

Arabidopsis, rice, wheat, Glycine max and Brassica rapa (Chang et al., 2016; Hu et al., 2018; Li

et al., 2019; Liu et al., 2019). These suggest that GhRbohs may play key roles in flower

development of cotton. The possible reasons for higher expression of GhRbohs in flowers might

be that: 1) timing flowering was essential for cotton acclimation to adverse stresses, which

requires more sensitive and complex ROS signal systems; 2) cotton undergoes long-term human

selection for high production of fibers, and for great tolerance to stress conditions, which are

closely related to flowering.

The effects of ABA, salinity, osmotic stress or heat stress on the transcript abundances of 26

GhRbohs were studied. The results revealed that different GhRbohs exhibited very diverse

expression patterns in responding to $\mathrm{ABA}$ and other abiotic stresses over time (Fig. 7-10, Table

S3-6), suggesting that each of the GhRbohs likely have differential and/or specific functions.

These GhRbohs may also be spatio-temporally controlled to generate special ROS signals in the

presence of ABA or under distinct stresses in cotton. The expression profiles of GhRbohs were

similar to those of Arabidopsis AtRbohs and rice OsRbohs in response to drought (PEG), salt and

heat stress (Chang et al, 2016), and those of soybean GmRbohs after treatment with salt or

osmotic stress (Liu et al, 2019), as well as those of Triticum aestivum TaRbohs in responding to

Peer) reviewing PDF | (2019:08:39951:2:0:NEW 8 Dec 2019) 
401

402

403

404

405

406

407

408

409

410

411

412

413

414

415

416

417

418

419

420

421

drought and heat stress (Navathe et al, 2019), reflecting the great diversity of the expression of Rbohs in plants under stresses.

Transcriptome analysis indicated that multiple genes like GhRbohK-1D, GhRbohK-2A, GhRbohK-2D, GhRbohD-3A and GhRbohF-3D specifically expressed at some stages of ovule growth and fiber development (Fig. 11), implying that these genes may be of very importance for fiber initiation and elongations through producing ROS in cotton.

Stressful conditions like high salinity, drought and high temperature as well as high concentrations of ABA commonly inhibit seedling growth, and promote flowering in plants including cotton (Takeno, 2016). The stressors and ABA also stimulate the expression of some GhRbohs (Fig. 7-10, Table S3-6), and cause the production of ROS in cotton. Therefore, GhRbohs-dependent ROS accumulation induced by the stresses or ABA probably play important roles in the modulation of cotton flowering and subsequent fiber development. ROS derived from NADPH oxidases has shown to act as signal molecules and positively modulate plant responses to diverse stresses (Suzuki et al., 2011; Marino et al., 2012; Chen and Yang, 2019). Environmental stressors generally suppress cotton fiber growth. It seems that ROS generated by the stressors and $\mathrm{ABA}$ inhibit fiber elongation. However, cotton has been under long term human selection for both high fiber yields and high tolerance to various stresses. It is conceivable that some stress-affected GhRbohs (most likely GhRbohKs, GhRbohDs and GhRbohFs) may be differentiated in functions and have the ability to positively regulate both fiber development and stress tolerance during the processes of natural evolution and human selection. Some stresscontrolled GhRbohs might also exist to negative influence fiber formation in cotton. Yet, the 
422 detailed mechanisms need to be thoroughly investigated in the future.

\section{CONCLUSIONS}

424 A total of 13, 13, 26 and 19 Rbohs were identified in G. arboretum, G. raimondii, G. hirsutum

425 and G. barbadense, respectively. These Rbohs were conserved in physical properties,

426 architectures of genes and motifs. The expansion of the Rbohs mainly relied on segmental

427 duplication, and was under the purifying selection. Most GhRbohs were highly expressed in

428 flowers. Different GhRbohs had very diverse expression patterns in responding to ABA, high

429 salinity, osmotic stress and heat stress. Some GhRbohs were preferentially and specifically

430 expressed during the development of ovules and fibers. Our results gave the comprehensive

431 information of the Gossypium Rbohs for further research towards understanding the roles of

432 Rbohs in cotton.

433

434 ADDITIONAL INFORMATION AND DECLARATIONS

435

436 Funding

437 This work was supported by the Fund of the State Key Laboratory of Cotton Biology, the

438 National Natural Science Foundation of China (31870248), the "111" Project, Science and

439 technology projects in Henan Province (192102110184) and Foundation of scientific research

440 and innovation team in Henan University of Animal Husbandry and Economy (2018KYTD18).

\section{Grant Disclosures}


443 The following grant information was disclosed by the authors:

444 Fund of the State Key Laboratory of Cotton Biology, the National Natural Science Foundation of

445 China: 31870248 .

446 "111" Project.

447 Science and technology projects in Henan Province: 192102110184.

448 Scientific Research and Innovation Team in Henan University of Animal Husbandry and

449 Economy: 2018KYTD18.

450

451 Competing Interests

452 The authors declare that they have no competing interests.

453

454

455

456

457 and approved the final version of the manuscript.

458

459

Figure 1 Phylogeny, gene arrangements and conserved motifs of Rboh members in four

\section{Authors' contributions}

FH designed the experiments and wrote the manuscript. GZ and LS conducted the experiments, CY and TL prepared the materials and finish all the bioinformatics work, All authors have read

\section{Conflict of Interest statement}

The authors declare no conflict of interest.

\section{Figure Legends}

Gossypium species. (A) The phylogenetic tree, (B) Exon/intron structures, the solid black boxes 
466 lines are exons and introns, respectively, (C) Profiles of the conserved motifs.

467 Figure 2 Distributions of Rbohs from G. arboretum (GaRbohs), G. raimondii (GrRbohs), G. 468 hirsutum (GhRbohs) and G. barbadense (GbRbohs) on chromosomes.

469 Figure 3 Synteny analysis of Rbohs of G. arboreum, G. raimondii, and G. hirsutum. Green 470 lines indicate gene pairs between G. arboreum and G. raimondii, red lines connected gene pairs between $G$. raimondii and $G$. hirsutum, blue lines show gene pairs between $G$. arboreum and $G$. hirsutum, yellow lines reveal gene pairs within individual species in G. arboreum, G. raimondii, 473 and $G$. hirsutum.

Figure 4 The $\mathrm{Ka} / \mathrm{Ks}$ results of the Rboh homologous genes between A genome, D genome and the subgenomes of $G$. hirsutum (AtDt).

Figure 5 The phylogenetic tree of Rbohs from four Gossypium species, and from A. thaliana,

T. cacao, R. communis, P. trichocarpa, G. max, B. distachyon, and O. sativa.

Figure 6 Expression profiles of 26 GhRbohs in distinct tissues of cotton. The genes are highly

expressed in flowers (A-X), and roots $(\mathrm{Y}-\mathrm{Z})$. The relative expression of the genes in roots was

set as 1 . Data are presented as mean $\pm \mathrm{SE}$. Different lowercase letters above the error bar indicate significant differences in gene expression levels among diverse tissues by one-way ANOVA and

Tukey's HSD test $(\mathrm{n} \geq 3, P \leq 0.05)$.

Figure 7 Effects of ABA on the expression of GhRbohs. The relative expression of GhRbohs was assayed after treatments with $100 \mu \mathrm{M}$ ABA for the indicated periods of time. The transcript abundances of the genes were rich at $0 \mathrm{~h}, 12 \mathrm{~h}$ and/or $24 \mathrm{~h}$ but few at $3 \mathrm{~h}$ and $6 \mathrm{~h}(\mathrm{~A}-\mathrm{F})$, were rich in $3 \mathrm{~h}$ and/or $6 \mathrm{~h}$, but small at other time (G-P), continually decreased (Q-W), and increased (X- 
487 Z). GhUBQ7 was act as the internal control. The expression value at $0 \mathrm{~h}$ was set as 1 . Data are

488 presented as mean \pm SE. Different lowercase letters above the error bar show significant

489 differences in gene expression levels at different time points by one-way ANOVA and Tukey's

490 HSD test $(\mathrm{n} \geq 3, P \leq 0.05)$.

491

492

493

494

495

496

497

498

499

500

501

502

503

504

505

506

507

Figure 8 Effects of $200 \mathrm{mM} \mathrm{NaCl}$ on the expression of GhRbohs. The transcription levels of the genes elevated $0 \mathrm{~h}, 12 \mathrm{~h}$ and/or $24 \mathrm{~h}$ but diminished at $3 \mathrm{~h}$ and $6 \mathrm{~h}(\mathrm{~A}-\mathrm{D})$, increased in $3 \mathrm{~h}$ and/or 6 h, but decreased at other time (E-P), continually reduced (Q-R), and enhanced (S-Z).

$G h U B Q 7$ was used as the internal control. The expression value at $0 \mathrm{~h}$ was set as 1 . Results are presented as mean \pm SE. Different lowercase letters above the error bar represent significant differences in transcript abundances at different time points by one-way ANOVA and Tukey's HSD test $(\mathrm{n} \geq 3, P \leq 0.05)$.

Figure 9 Effects of 10\% PEG on the expression of GhRbohs. The expression levels of the GhRbohs increased at $0 \mathrm{~h}, 12 \mathrm{~h}$ and/or $24 \mathrm{~h}$ but dropped at $3 \mathrm{~h}$ and $6 \mathrm{~h}(\mathrm{~A}-\mathrm{J})$, heightened in $3 \mathrm{~h}$ and/or $6 \mathrm{~h}$, but cut down at other time (K-Q), continually decreased (R-U), and increased (V-Z). GhUBQ7 was applied as the internal control. The expression value at $0 \mathrm{~h}$ was set as 1 . Data are presented as mean \pm SE. Different lowercase letters above the error bar indicate significant differences in gene transcription levels at different time points by one-way ANOVA and Tukey's HSD test $(\mathrm{n} \geq 3, P \leq 0.05)$.

Figure 10 The expression profiles of GhRbohs under high temperature stress. The expression of the GhRbohs upregulated at $0 \mathrm{~h}, 12 \mathrm{~h}$ and/or $24 \mathrm{~h}$ but downregulated at $3 \mathrm{~h}$ and $6 \mathrm{~h}$ (A-E), increased at $3 \mathrm{~h}$ and/or $6 \mathrm{~h}$, but reduced at other time $(\mathrm{F}-\mathrm{W})$, continually lowered $(\mathrm{X}-\mathrm{Y})$, 
508

509

510

511

512

513

514

515

516

517

518

519

520

521

522

523

524

525

526

527

528

and elevated (Z). GhUBQ7 acts as the internal control. The relative expression value at $0 \mathrm{~h}$ was set as 1. Data are presented as mean \pm SE. Different lowercase letters above the error bar show significant differences in gene expression levels at different time points by one-way ANOVA and Tukey's HSD test $(\mathrm{n} \geq 3, P \leq 0.05)$.

Figure 11 Changes in expression of GhRbohs during development of ovules and fibers in cotton. The heat maps in yellow/white/blue (from low to high) indicate the expression levels of various GhRbohs at different stages of fiber initiation and elongation.

\section{References}

Allen RD. 2010. Opportunities for engineering abiotic stress tolerance in cotton plants. In: Cotton. Berlin: Springer Verlag, 127-160.

Camacho C, Coulouris G, Avagyan V, Ma N, Papadopoulos J, Bealer K, Madden LT. 2009. Blast+: architecture and applications. BMC Bioinformatics 10:421.

Cannon SB, Mitra A, Baumgarten A, Young ND, May G. 2004. The roles of segmental and tandem gene duplication in the evolution of large gene families in Arabidopsis thaliana. BMC Plant Biology 4:10 DOI 10.1186/1471-2229-4-10.

\section{Chang YL, Li WY, Miao H, Yang SQ, Li R, Wang X, Li WQ, Chen KM. 2016.} Comprehensive genomic analysis and expression profiling of the NOX gene families under abiotic stresses and hormones in plants. Genome Biology and Evolution 8(3):791-810 DOI 10.1093/gbe/evw035.

Chen CJ, Xia R, Chen H, He YH. 2018. TBtools, a toolkit for biologists integrating various 
529

530

531

532

533

534

535

536

537

538

539

540

541

542

543

544

545

546

547

548

549

HTS-data handling tools with a user-friendly interface. BioRxiv, 289660 DOI

10.1101/289660.

Cheng X, Li G, Manzoor MA, Wang H, Abdullah M, Su X, Zhang J, Jiang T, Jin Q, Cai Y, Lin Y. 2019. In Silico Genome-Wide analysis of Respiratory Burst Oxidase Homolog $(\mathrm{RBOH})$ family genes in five fruit-producing trees, and potential functional analysis on lignification of stone cells in chinese White Pear. Cells 8:520 DOI 10.3390/cells8060520.

Chen Q, Yang G. 2019. Signal function studies of ROS, especially RBOH-dependent ROS, in plant growth, development and environmental stress. Journal of Plant Growth Regulation, 1-15 DOI 10.1007/s00344-019-09971-4.

Foreman J, Demidchik V, Bothwell JHF, Mylona P, Miedema H, Torres MA, Linstead P, Costa S, Brownlee C, Jones JDG, Davies JM, Dolan L. 2003. Reactive oxygen species produced by NADPH oxidase regulate plant cell growth. Nature 422:442-446 DOI 10.1038 /nature 01485 .

He H, Yan JW, Yu XY, Liang Y, Fang L, Scheller HV, Zhang AY. 2017. The NADPHoxidase AtRbohI plays a positive role in drought-stress response in Arabidopsis thaliana. Biochemical and Biophysical Research Communications 491(3):834-839 DOI 10.1016/j.bbrc.2017.05.131.

Hu CH, Wei XY, Yuan B, Yao LB, Ma TT, Zhang PP, Wang X, Wang PQ, Liu WT, Li WQ, Meng LS, Chen KM. 2018. Genome-Wide identification and functional analysis of NADPH oxidase family genes in Wheat during development and environmental stress responses. Frontiers in Plant Science, 9-906 DOI 10.3389/fpls.2018.00906. 
550

551

552

553

554

555

556

557

558

559

560

561

562

563

564

565

566

567

568

569

570

Jiao YH, Sun LR, Song YL, Wang LM, Liu LP, Zhang LY, Liu B, Li N, Miao C, Hao FS. 2013. AtrbohD and AtrbohF positively regulate abscisic acid inhibited primary root growth by affecting $\mathrm{Ca} 2+$ signaling and auxin response of roots in Arabidopsis. Journal of Experimental Botany 64:4183-4192 DOI 10.1093/jxb/ert228.

Kalyaanamoorthy S, Minh BQ, Wong TKF, Von Haeseker A, Jermiin LS. 2017. ModelFinder: fast model selection for accurate phylogenetic estimates. Nature Methods 14(6):587-589 DOI 10.1038/nmeth.4285.

Kaya H, Nakajima R, Iwano M, Kanaoka MM, Kimura S, Takeda S, Kawarazak T, Senzak E, Kuchitsu K. 2014. $\mathrm{Ca}^{2+}$-activated reactive oxygen species production by Arabidopsis $\mathrm{RbohH}$ and RbohJ is essential for proper pollen tube tip growth. Plant Cell 26: 1069-1080 DOI 10.1105/tpc.113.120642.

Li D, Wu D, Li S, Dai Y, Cao Y. 2019. Evolutionary and functional analysis of the plantspecific NADPH oxidase gene family in Brassica rapa L. Royal Society Open Science 6:181727 DOI 10.1098/rsos.181727.

Li N, Sun LR, Zhang LY, Song YL, Hu PP, Li C, Hao FS. 2015. AtrbohD and AtrbohF negatively regulate lateral root development by changing the localized accumulation of superoxide in primary roots of Arabidopsis. Planta 241:591-602 DOI 10.1007/s00425-0142204-1.

Li PT, Rashid MH, Chen TT, Lu QW, Ge Q, Gong WK, Liu AY, Gong JW, Shang HH, Deng XY, Li JW, Li SQ, Xiao XH, Liu RX, Zhang Q, Duan L, Zou XY, Zhang Z, Jiang X, Zhang Y, Peng RH, Shi YZ, Yuan YL. 2019. Transcriptomic and biochemical 
analysis of upland cotton (Gossypium hirsutum) and a chromosome segment substitution line from G. hirsutum $\times$ G. barbadense in response to Verticillium dahliae infection. BMC Plant Biology. 19:19 DOI 10.1186/s12870-018-1619-4.

Liu B, Sun LR, Ma LY, Hao FS. 2017. Both AtrbohD and AtrbohF are essential for mediating responses to oxygen deficiency in Arabidopsis. Plant Cell Reports 36:947-957 DOI $10.1007 / \mathrm{s} 00299-017-2128-\mathrm{x}$.

Liu J, Lu H, Wan Q, Qi W, Shao H. 2019. Genome-wide analysis and expression profiling of respiratory burst oxidase homologue gene family in Glycine max. Environmental and Experimental Botany 161:344-356 DOI 10.1016/j.envexpbot.2018.07.015.

Lu TT, Zhang GF, Sun LR, Wang J, Hao FS. 2017. Genome-wide identification of CBL family and expression analysis of CBLs in response to potassium deficiency in cotton. PeerJ 5:e3653 DOI 10.7717/peerj.3653

Lu TT, Zhang GF, Wang YB, He SB, Sun LR, Hao FS. 2019. Genome-wide characterization and expression analysis of PP2CA family members in response to ABA and osmotic stress in Gossypium. PeerJ 7:e7105 DOI 10.7717/peerj.7105.

Macpherson N, Takeda S, Shang Z, Dark A, Mortimer JC, Brownlee C, Dolan L, Davies JM. 2008. NADPH oxidase involvement in cellular integrity. Planta 227:1415-1418 DOI 10.1007/s00425-008-0716-2.

Ma LY, Zhang H, Sun LR, Jiao YH, Zhang GZ, Miao C, Hao FS. 2012. NADPH oxidase AtrbohD and AtrbohF function in ROS-dependent regulation of $\mathrm{Na}^{+} / \mathrm{K}^{+}$homeostasis in Arabidopsis under salt stress. Journal of Experimental Botany 63:305-317 DOI 
592

593

594

595

596

597

598

599

600

601

602

603

604

605

606

607

608

609

610

611

612

$10.1093 / \mathrm{jxb} / \mathrm{err} 280$.

Mangano S, Denita-Juarez SP, Choi HS, Marzol E, Hwang Y, Ranocha P, Muschietti JP. 2017. Molecular link between auxin and ROS-mediated polar growth. Proceedings of the National Academy of Sciences of the United States of America 114(20):5289-5294 DOI $10.1073 /$ pnas. 1701536114 .

Marino D, Dunand C, Puppo A, Pauly N. 2012. A burst of plant NADPH oxidases. Trends in Plant Science 17(1):9-15 DOI 10.1016/j.tplants.2011.10.001.

Mhamdi A, Breusegem FV. 2018. Reactive oxygen species in plant development. Development 145:dev164376 DOI 10.1242/dev.164376.

Minh BQ, Nguyen MA, Von Haeseler A. 2013. Ultrafast approximation for phylogenetic bootstrap. Molecular Biology and Evolution 30:1188-1195 DOI 10.1093/molbev/mst024.

Mohanta TK, Mohanta N, Mohanta YK, Parida P, Bae H. 2015. Genome-wide identification of calcineurin B-Like (CBL) gene family of plants reveals novel conserved motifs and evolutionary aspects in calcium signaling events. BMC Plant Biology 15:189 DOI 10.1186/s12870-015-0543-0.

Müller K, Carstens AC, Linkies A, Torres MA, Leubner-Metzger G. 2009. The NADPHoxidase AtrbohB plays a role in Arabidopsis seed after-ripening. New Phytologist 184:885897 DOI 10.1111/j.1469-8137.2009.03005.x.

Navathe S, Singh S, Singh VK, Chand R, Mishra VK, Joshi AK. 2019. Genome-wide mining of respiratory burst homologs and its expression in response to biotic and abiotic stresses in Triticum aestivum. Genes \& Genomics, 1-17 DOI 10.1007/s13258-019-00821-x. 
613 Nguyen LT, Schmidt HA, Von Haeseler A, Minh BQ. 2015. IQ-TREE: a fast and effective

614

615

616

617

618

619

620

621

622

623

624

625

626

627

628

629

630

631

632

633

stochastic algorithm for estimating maximum-likelihood phylogenies. Molecular Biology and Evolution 32(1):268-274 DOI 10.1093/molbev/msu300.

Potikha TS, Collins CC, Johnson DI, Delmer DP, Levine A. 1999. The involvement of hydrogen peroxide in the differentiation of secondary walls in cotton fibers. Plant Physiology 119(3):849-858 DOI 10.1104/pp.119.3.849.

Qi J, Song CP, Wang B, Zhou J, Kangasjärvi J, Zhu JK, Gong Z. 2018. Reactive oxygen species signaling and stomatal movement in plant responses to drought stress and pathogen attack. Journal of Integrative Plant Biology 60(09):67-88 DOI 10.1111/jipb.12654.

Song Y, Miao Y, Song CP. 2014. Behind the scenes: the roles of reactive oxygen species in guard cells. New Phytologist 201(4):1121-1140 DOI 10.1111/nph.12565.

Sun LR, Ma LY, He SB, Hao FS. 2018. AtrbohD functions downstream of ROP2 and positively regulates waterlogging response in Arabidopsis. Plant Signaling \& Behavior 13:9-e1513300 DOI 10.1080/15592324.2018.1513300.

Sun LR, Zhao ZJ, Hao FS. 2019. NADPH oxidases, essential players of hormone signalings in plant development and response to stresses. Plant Signaling \& Behavior. 14:1657343.

Suzuki N, Miller G, Morales J, Morales J, Shulaev V, Torres MA, Mittler R. 2011. Respiratory burst oxidases: the engines of ROS signaling. Current Opinion in Plant Biology 14(6):691-699 DOI 10.1016/j.pbi.2011.07.014.

Takeno, K., 2016. Stress-induced flowering: the third category of flowering response. Journal of Experimental Botany. 67:4925-4934 DOI 10.1093/jxb/erw272. 
634 Wang F, Chen ZH, Liu X, Shabala L, Yu M, Zhou M, Salih A, Shabala S. 2019. The loss of 635 RBOHD function modulates root adaptive responses to combined hypoxia and salinity 636 stress in Arabidopsis. Environmental and Experimental Botany. 158:125-135 DOI 10.1016/j.envexpbot.2018.11.020.

638 639

Xia XJ, Zhou YH, Shi K, Zhou J, Foyer CH, Yu JQ. 2015. Interplay between reactive oxygen species and hormones in the control of plant development and stress tolerance. Journal of Experimental Botany 66(10):2839-2856 DOI 10.1093/jxb/erv089.

Xie HT, Wan ZY, Li S, Zhang Y. 2014. Spatiotemporal production of reactive oxygen species by NADPH oxidase is critical for tapetal programmed cell death and pollen development in Arabidopsis. Plant Cell 26:2007-2023 DOI 10.1105/tpc.114.125427.

Zhang GF, Lu TT, Miao WW, Sun LR, Tian M, Wang J, Hao FS. 2017. Genome-wide identification of ABA receptor PYL family and expression analysis of PYLs in response to ABA and osmotic stress in Gossypium. PeerJ 5:e4126 DOI 10.7717/peerj.4126.

Zou Z, Yang J, Zhang X. 2019. Insights into genes encoding respiratory burst oxidase homologs (RBOHs) in rubber tree (Hevea brasiliensis Muell. Arg.). Industrial Crops and Products 128:126-139 DOI 10.1016/j.indcrop.2018.11.005. 
Table $\mathbf{1}$ (on next page)

Rboh family members in Gossypium 


\begin{tabular}{|c|c|c|c|c|}
\hline Gene identifier & Gene name & $\begin{array}{l}\text { Size } \\
\text { (AA) }\end{array}$ & $\begin{array}{l}\text { Mass } \\
(\mathrm{kDa})\end{array}$ & $\mathrm{pI}$ \\
\hline Cotton_A_29320 & GaRbohB & 884 & 100.8 & 9.19 \\
\hline Cotton_A_13752 & GaRbohD-1 & 933 & 105.3 & 9.04 \\
\hline Cotton_A_33534 & GaRbohD-2 & 916 & 104.1 & 9.13 \\
\hline Cotton_A_18772 & GaRbohD-3 & 872 & 98.6 & 9.12 \\
\hline Cotton_A_05673 & GaRbohE & 1015 & 115.5 & 9.1 \\
\hline Cotton_A_38536 & GaRbohF-1 & 929 & 106 & 9.28 \\
\hline Cotton_A_31123 & GaRbohF-2 & 871 & 99.8 & 9.09 \\
\hline Cotton_A_23171 & GaRbohF-3 & 846 & 95.7 & 9.12 \\
\hline Cotton_A_24667 & GaRbohH & 1273 & 145 & 8.94 \\
\hline Cotton_A_03631 & GaRbohK-1 & 907 & 102.9 & 9.23 \\
\hline Cotton_A_07974 & GaRbohK-2 & 915 & 103.6 & 9.17 \\
\hline Cotton_A_16216 & GaRbohK-3 & 919 & 104 & 8.82 \\
\hline Cotton_A_13344 & GaRbohL & 803 & 91.1 & 9.08 \\
\hline Gorai.007G299500 & GrRbohB & 884 & 100.9 & 9.19 \\
\hline Gorai.009G202500 & GrRbohD-1 & 936 & 105.4 & 9.03 \\
\hline Gorai.002G128200 & GrRbohD-2 & 786 & 89 & 8.94 \\
\hline Gorai.009G273400 & GrRbohD-3 & 930 & 104.7 & 9.13 \\
\hline Gorai.001G017400 & GrRbohE & 943 & 107 & 8.87 \\
\hline Gorai.003G085100 & GrRbohF-1 & 929 & 105.9 & 9.3 \\
\hline Gorai.004G137300 & GrRbohF-2 & 921 & 105.1 & 9.32 \\
\hline Gorai.008G250500 & GrRbohF-3 & 928 & 105.8 & 9.35 \\
\hline
\end{tabular}




\begin{tabular}{|c|c|c|c|c|}
\hline Gorai.001G106500 & GrRbohH & 841 & 96 & 9.1 \\
\hline Gorai.001G053300 & GrRbohK-1 & 907 & 102.7 & 9.07 \\
\hline Gorai.008G212100 & GrRbohK-2 & 920 & 104.4 & 9.02 \\
\hline Gorai.003G117900 & GrRbohK-3 & 919 & 103.7 & 8.9 \\
\hline Gorai.008G199100 & GrRbohL & 802 & 90.7 & 9.11 \\
\hline Gh_A11G2426 & GhRbohBA & 884 & 100.7 & 9.22 \\
\hline Gh_D11G2743 & GhRbohBD & 884 & 100.8 & 9.19 \\
\hline Gh_A05G1666 & GhRbohD-1A & 721 & 81.2 & 9.29 \\
\hline Gh_D05G1864 & GhRbohD-1D & 913 & 102.6 & 9.07 \\
\hline Gh_A01G0943 & GhRbohD-2A & 857 & 97 & 8.84 \\
\hline Gh_D01G0990 & GhRbohD-2D & 940 & 107.1 & 8.97 \\
\hline Gh_A05G2211 & GhRbohD-3A & 849 & 94.8 & 9.19 \\
\hline Gh_D05G2471 & GhRbohD-3D & 932 & 105 & 9.06 \\
\hline Gh_A07G0143 & GhRbohEA & 753 & 85.6 & 9.18 \\
\hline Gh_D07G0136 & GhRbohED & 753 & 85.6 & 9.14 \\
\hline Gh_A02G1791 & GhRbohF-1A & 930 & 106 & 9.28 \\
\hline Gh_D03G0688 & GhRbohF-1D & 929 & 105.9 & 9.33 \\
\hline Gh_A08G0982 & GhRbohF-2A & 751 & 86.5 & 9.63 \\
\hline Gh_D08G1257 & GhRbohF-2D & 922 & 105.2 & 9.28 \\
\hline Gh_A12G2669 & GhRbohF-3A & 929 & 105.8 & 9.29 \\
\hline Gh_D12G2750 & GhRbohF-3D & 928 & 105.7 & 9.35 \\
\hline Gh_A07G0856 & GhRbohHA & 841 & 95.9 & 9.01 \\
\hline Gh_D07G0928 & GhRbohHD & 841 & 95.9 & 9.07 \\
\hline
\end{tabular}




\begin{tabular}{|c|c|c|c|c|}
\hline Gh_A07G0398 & GhRbohK-1A & 907 & 102.9 & 9.23 \\
\hline Gh_D07G0463 & GhRbohK-1D & 907 & 102.7 & 9 \\
\hline Gh_A12G1774 & GhRbohK-2A & 918 & 104 & 9.16 \\
\hline Gh_D12G1932 & GhRbohK-2D & 918 & 104.2 & 9.18 \\
\hline Gh_A03G0476 & GhRbohK-3A & 904 & 102.7 & 8.65 \\
\hline Gh_D03G1062 & GhRbohK-3D & 919 & 103.8 & 8.82 \\
\hline Gh_A12G2653 & GhRbohLA & 803 & 91.1 & 9.11 \\
\hline Gh_D12G1807 & GhRbohLD & 802 & 90.8 & 8.99 \\
\hline GOBAR_AA19255 & GbRbohBA & 884 & 100.8 & 9.22 \\
\hline GOBAR_DD12974 & GbRbohBD & 884 & 100.8 & 9.19 \\
\hline GOBAR_AA06210 & GbRbohD-1A & 933 & 105.2 & 8.96 \\
\hline GOBAR_AA29299 & GbRbohD-2A & 942 & 107.3 & 9.13 \\
\hline GOBAR_DD22521 & GbRbohD-2D & 915 & 104.2 & 9.03 \\
\hline GOBAR_AA19212 & GbRbohEA & 951 & 108 & 9.05 \\
\hline GOBAR_DD05976 & GbRbohED & 943 & 107 & 8.87 \\
\hline GOBAR_AA24991 & GbRbohF-1A & 929 & 106 & 9.28 \\
\hline GOBAR_AA08909 & GbRbohF-2A & 878 & 99.6 & 8.91 \\
\hline GOBAR_AA23701 & GbRbohF-3A & 928 & 105.7 & 9.29 \\
\hline GOBAR_AA36532 & GbRbohHA & 872 & 99.9 & 9.15 \\
\hline GOBAR_DD21834 & GbRbohHD & 833 & 95.3 & 9.16 \\
\hline GOBAR_AA28632 & GbRbohK-1A & 880 & 99.7 & 9.41 \\
\hline GOBAR_DD31632 & GbRbohK-1D & 907 & 102.7 & 9.07 \\
\hline GOBAR_AA11222 & GbRbohK-2A & 839 & 95.5 & 9.23 \\
\hline
\end{tabular}




\begin{tabular}{llccc}
\hline GOBAR_DD20994 & GbRbohK-2D & 985 & 112 & 9.34 \\
GOBAR_AA01568 & GbRbohK-3A & 891 & 100.6 & 8.71 \\
GOBAR_DD02070 & GbRbohK-3D & 914 & 103.3 & 8.83 \\
GOBAR_DD30619 & GbRbohLD & 688 & 78.3 & 9.08 \\
\hline
\end{tabular}

1 
Figure 1

Phylogeny, gene arrangements and conserved motifs of Rboh members in four Gossypium species.

(A) The phylogenetic tree, (B) Exon/intron structures, the solid black boxes lines are exons and introns, respectively, (C) Profiles of the conserved motifs.

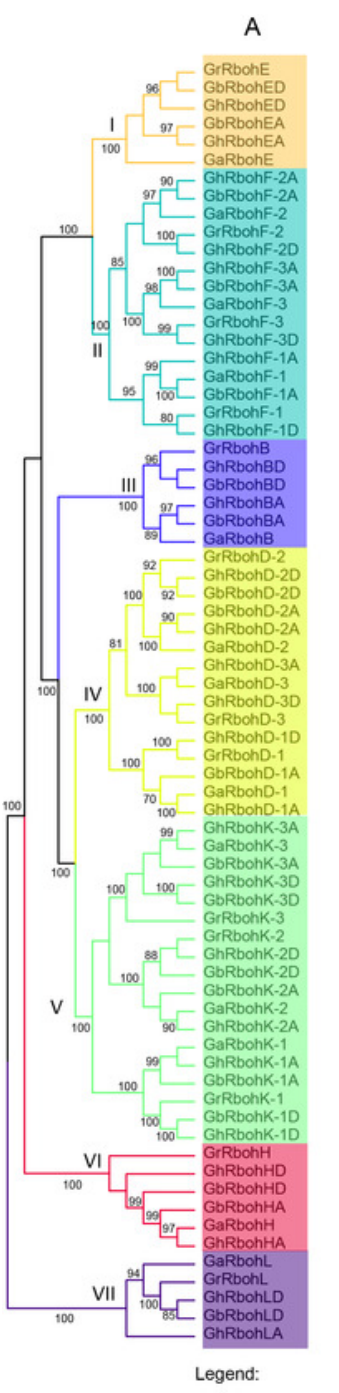

B
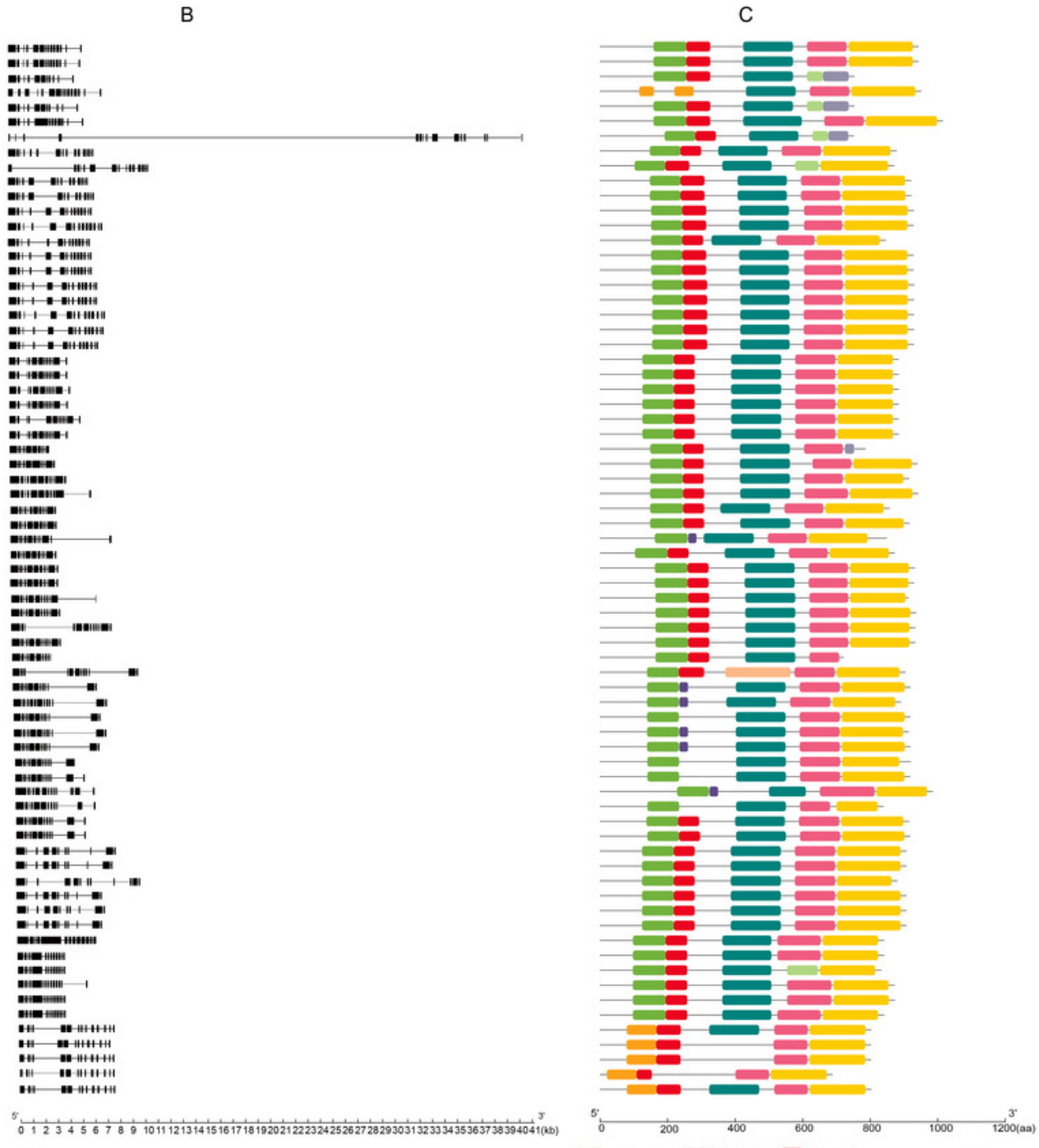

- NADPH_OX FAD_Binding_8 8 EF-hand_7 FNR_ike superfamily EF-hand_e

Exon -intron 
Figure 2

Distributions of Rbohs from G. arboretum (GaRbohs), G. raimondii (GrRbohs), G. hirsutum (GhRbohs) and G. barbadense (GbRbohs) on chromosomes. 


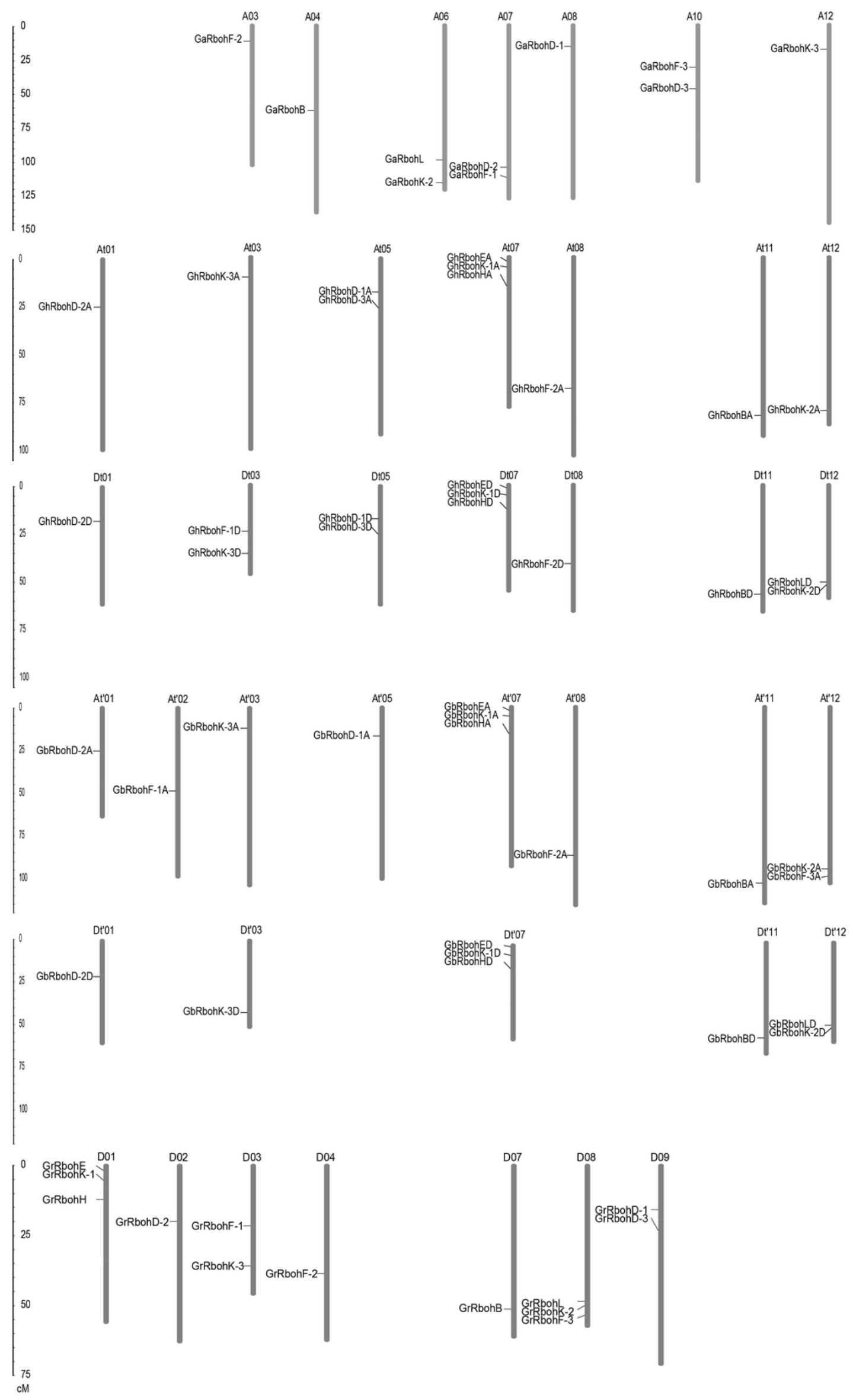

Peer] reviewing PDF | (2019:08:39951:2:0:NEW 8 Dec 2019) 
Figure 3

Synteny analysis of Rbohs of $G$. arboreum, G. raimondii, and $G$. hirsutum.

Green lines indicate gene pairs between $G$. arboreum and $G$. raimondii, red lines connected gene pairs between $G$. raimondii and $G$. hirsutum, blue lines show gene pairs between $G$. arboreum and $G$. hirsutum, yellow lines reveal gene pairs within individual species in $G$. arboreum, G. raimondii, and G. hirsutum. 


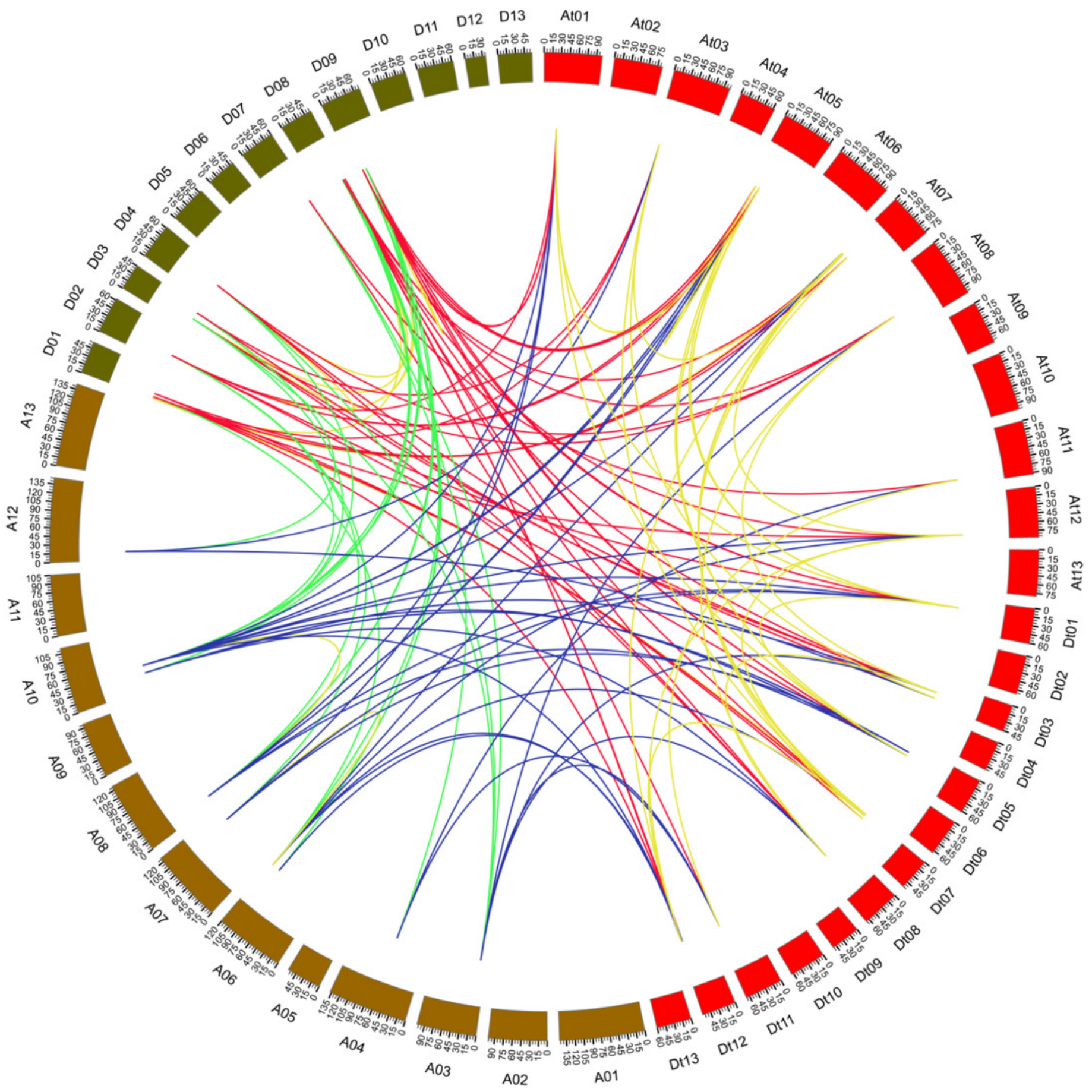


Figure 4

The $\mathrm{Ka} / \mathrm{Ks}$ results of the Rboh homologous genes between $\mathrm{A}$ genome, $\mathrm{D}$ genome and the subgenomes of $G$. hirsutum (AtDt).

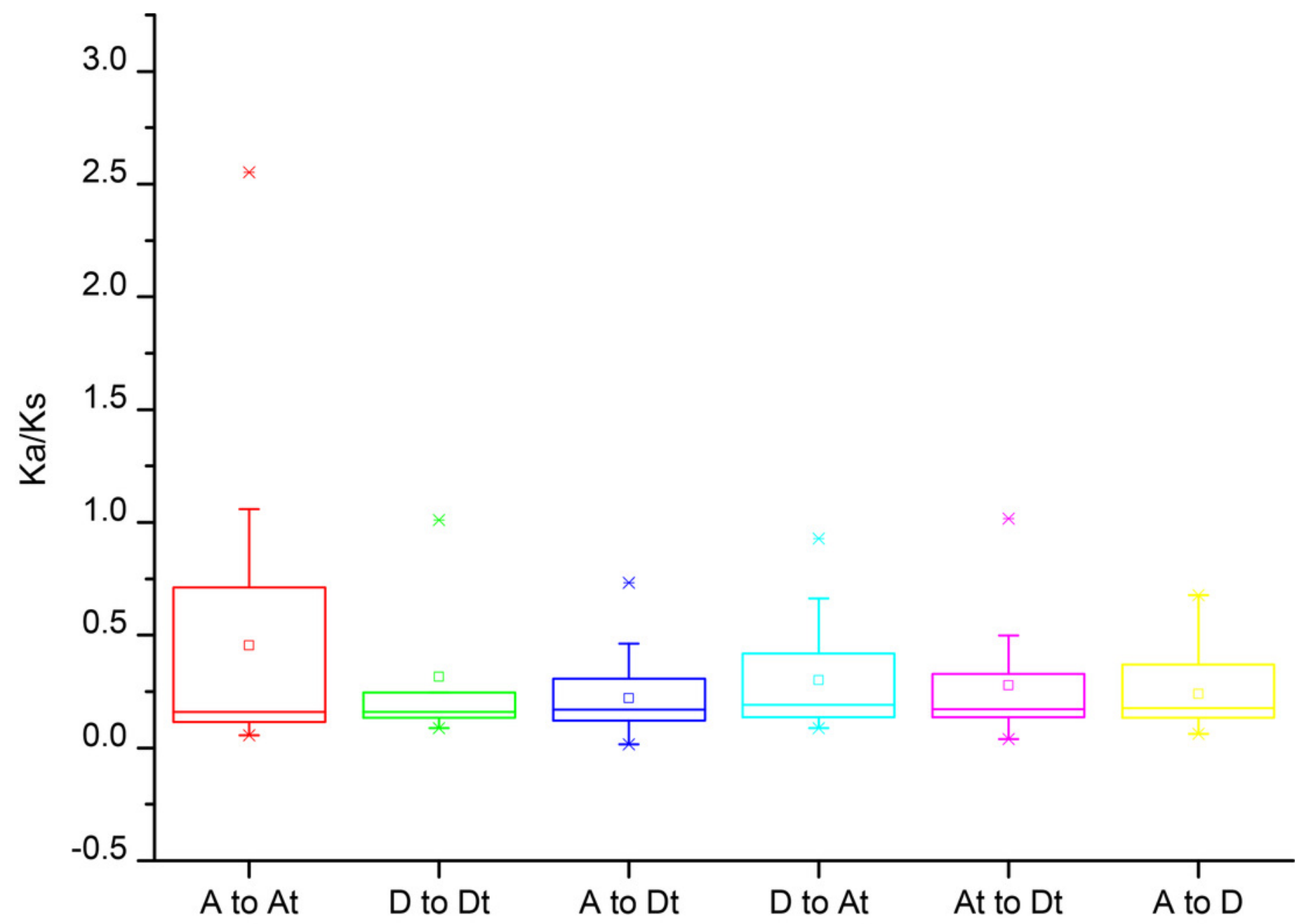




\section{Figure 5}

The phylogenetic tree of Rbohs from four Gossypium species, and from $A$. thaliana, $T$. cacao, R. communis, P. trichocarpa, G. max, B. distachyon, and $O$. sativa.

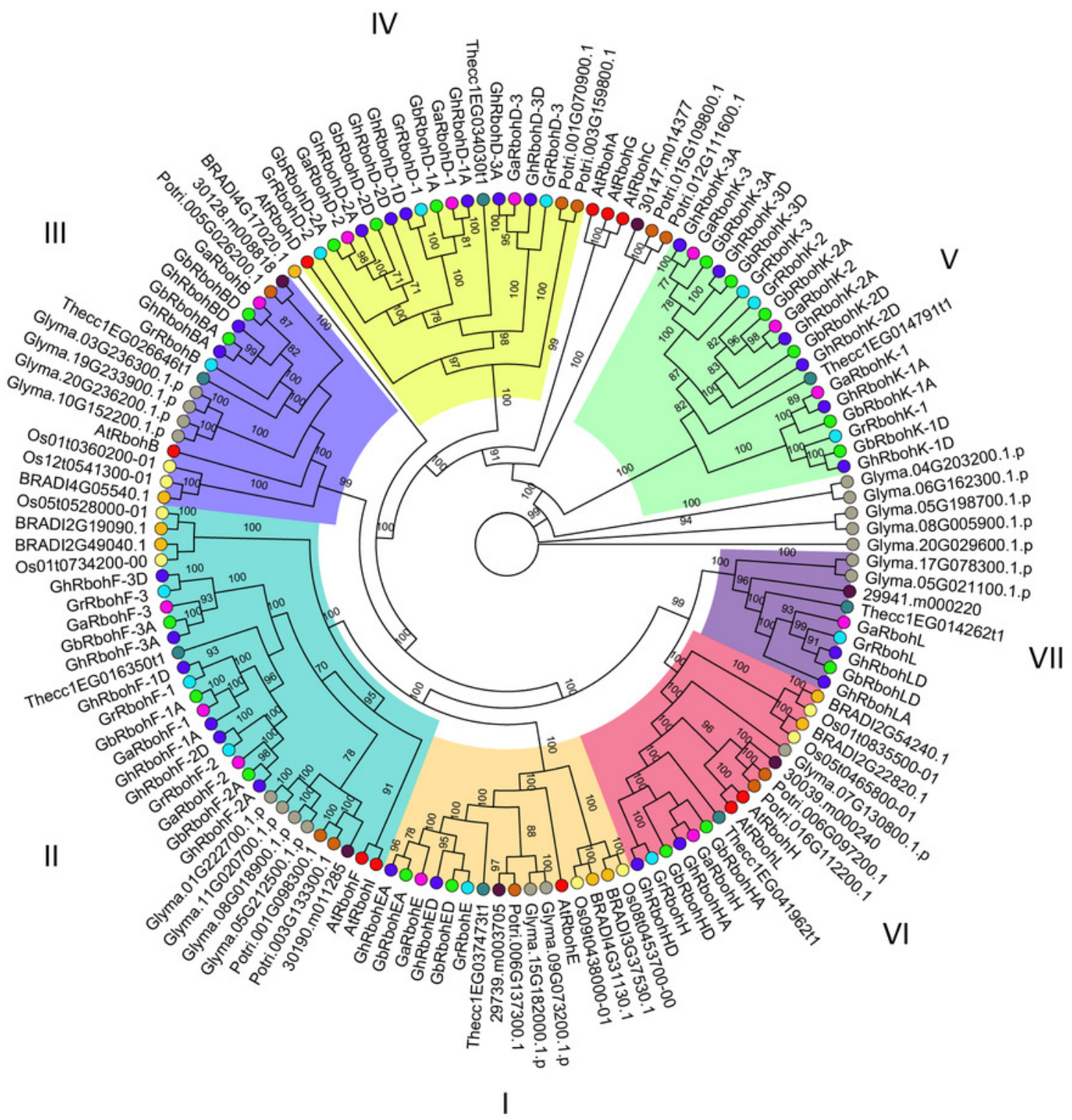

G. arboreum

G. raimondii

G. hirsutum

G. barbadense

A. thaliana

T. cacao

- R. communis

P. trichocarpa

G. max

B. distachyon

O. sativa 


\section{Figure 6}

Expression profiles of 26 GhRbohs in distinct tissues of cotton.

The genes are highly expressed in flowers (A-X), and roots (Y-Z). The relative expression of the genes in roots was set as 1 . Data are presented as mean \pm SE. Different lowercase letters above the error bar indicate significant differences in gene expression levels among diverse tissues by one-way ANOVA and Tukey's HSD test ( $n \geq 3, P \leq 0.05)$. 

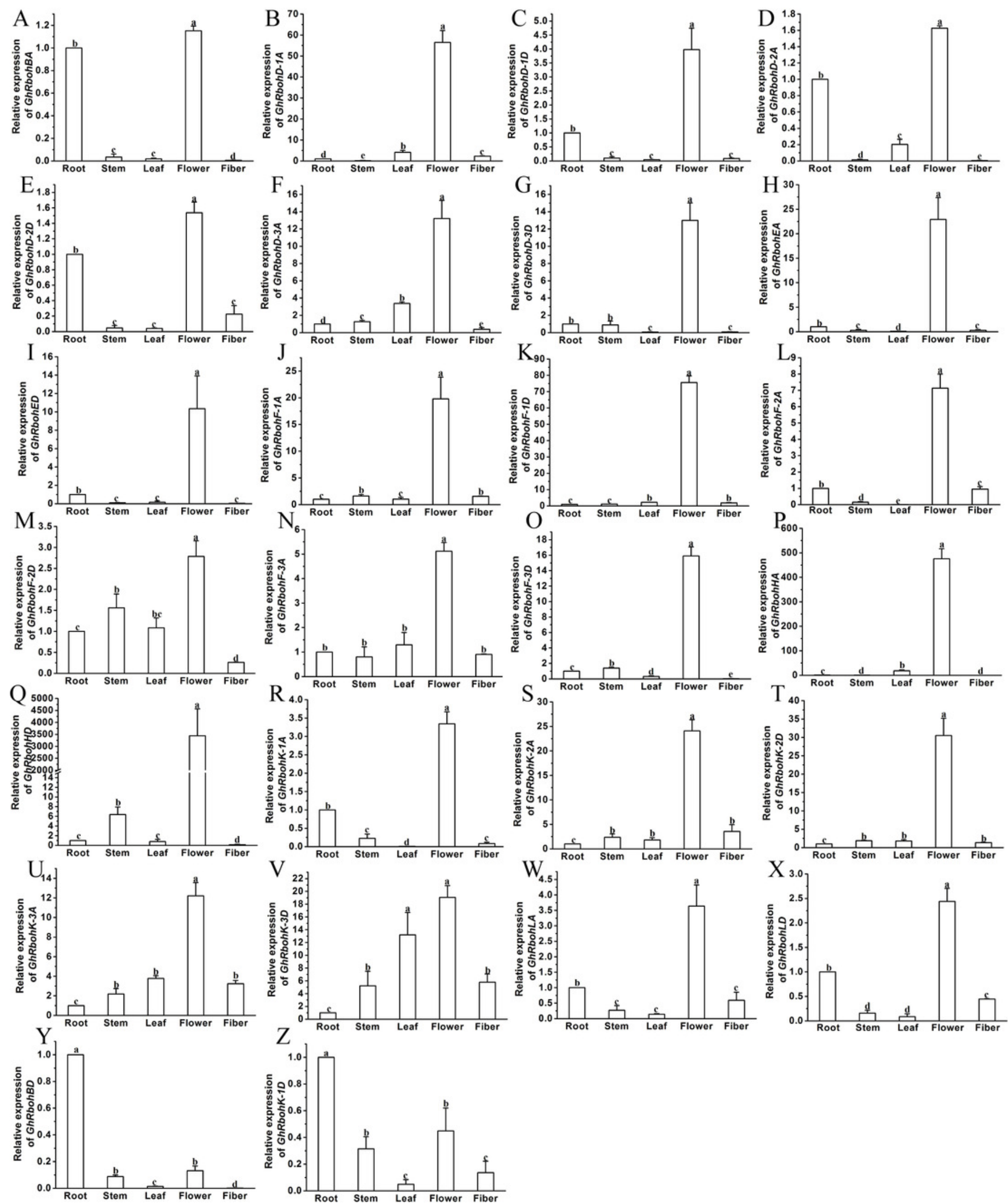


\section{Figure 7}

Effects of ABA on the expression of GhRbohs.

The relative expression of GhRbohs was assayed after treatments with $100 \mu \mathrm{M}$ ABA for the indicated periods of time. The transcript abundances of the genes were rich at $0 \mathrm{~h}, 12 \mathrm{~h}$ and/or $24 \mathrm{~h}$ but few at $3 \mathrm{~h}$ and $6 \mathrm{~h}(\mathrm{~A}-\mathrm{F})$, were rich in $3 \mathrm{~h}$ and/or $6 \mathrm{~h}$, but small at other time (G-P), continually decreased (Q-W), and increased (X-Z). GhUBQ7 was act as the internal control. The expression value at $0 \mathrm{~h}$ was set as 1 . Data are presented as mean $\pm \mathrm{SE}$. Different lowercase letters above the error bar show significant differences in gene expression levels at different time points by one-way ANOVA and Tukey's HSD test $(n \geq 3, P \leq$ $0.05)$. 

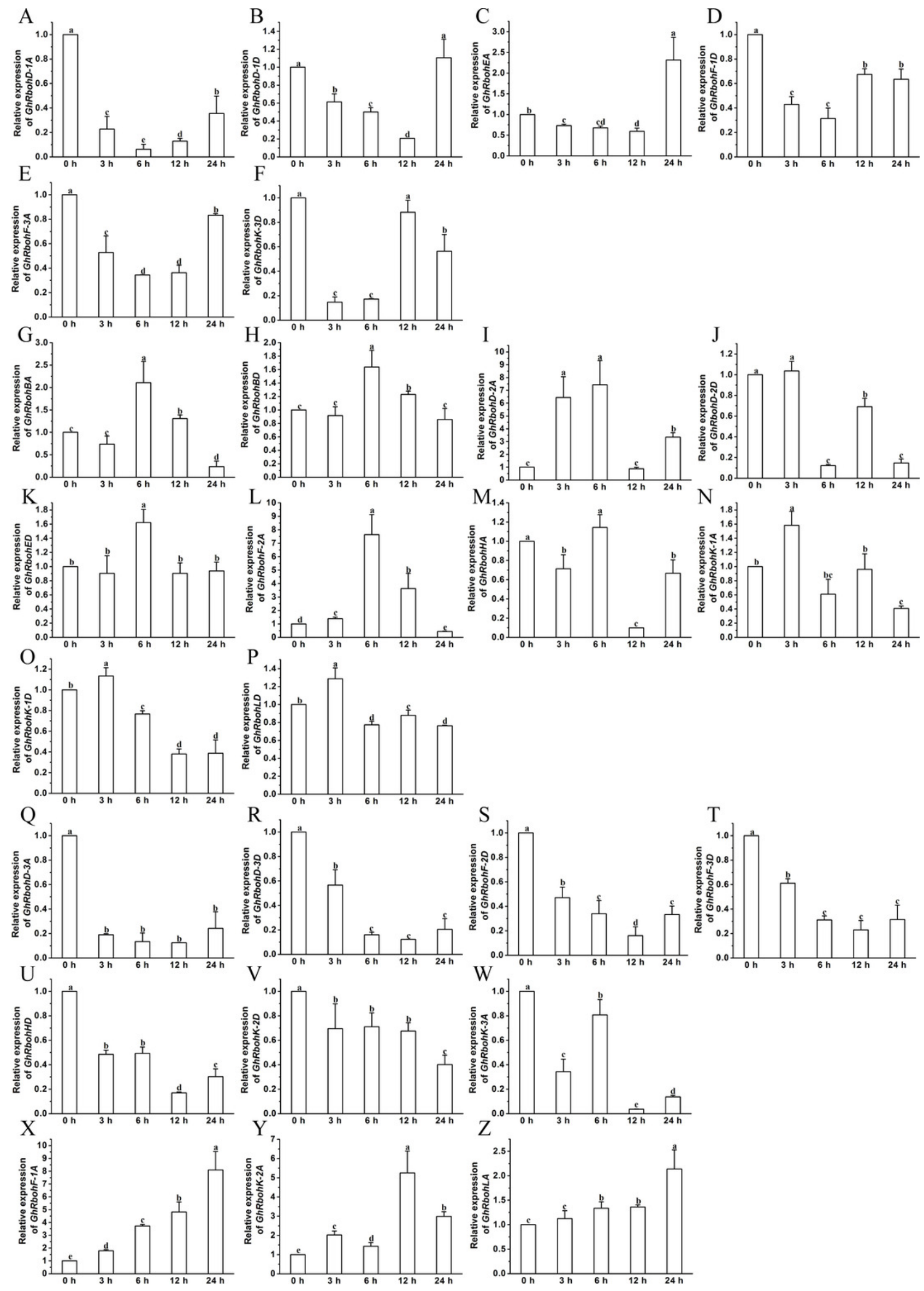

PeerJ reviewing PDF | (2019:08:39951:2:0:NEW 8 Dec 2019) 


\section{Figure 8}

Effects of $200 \mathrm{mM} \mathrm{NaCl}$ on the expression of GhRbohs.

The transcription levels of the genes elevated $0 \mathrm{~h}, 12 \mathrm{~h}$ and/or $24 \mathrm{~h}$ but diminished at $3 \mathrm{~h}$ and $6 \mathrm{~h}(\mathrm{~A}-\mathrm{D})$, increased in $3 \mathrm{~h}$ and/or $6 \mathrm{~h}$, but decreased at other time (E-P), continually reduced (Q-R), and enhanced (S-Z). GhUBQ7 was used as the internal control. The expression value at $0 \mathrm{~h}$ was set as 1 . Results are presented as mean $\pm \mathrm{SE}$. Different lowercase letters above the error bar represent significant differences in transcript abundances at different time points by one-way ANOVA and Tukey's HSD test ( $\mathrm{n} \geq 3, P \leq 0.05)$. 

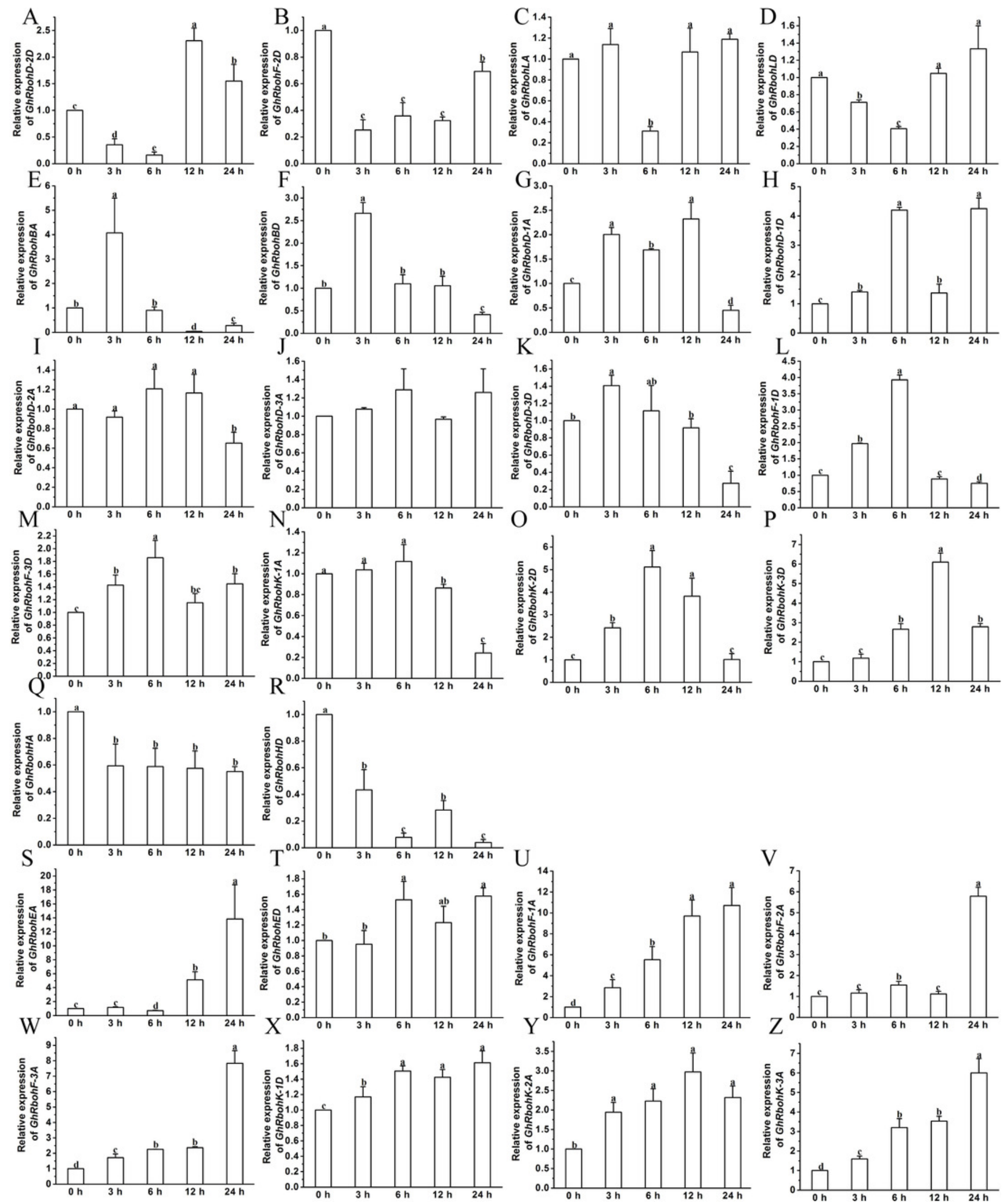
Figure 9

Effects of $10 \%$ PEG on the expression of GhRbohs.

The expression levels of the GhRbohs increased at $0 \mathrm{~h}, 12 \mathrm{~h}$ and/or $24 \mathrm{~h}$ but dropped at $3 \mathrm{~h}$ and $6 \mathrm{~h}(\mathrm{~A}-\mathrm{J})$, heightened in $3 \mathrm{~h}$ and/or $6 \mathrm{~h}$, but cut down at other time (K-Q), continually decreased (R-U), and increased (V-Z). GhUBQ7 was applied as the internal control. The expression value at $0 \mathrm{~h}$ was set as 1 . Data are presented as mean $\pm \mathrm{SE}$. Different lowercase letters above the error bar indicate significant differences in gene transcription levels at different time points by one-way ANOVA and Tukey's HSD test ( $n \geq 3, P \leq 0.05)$. 

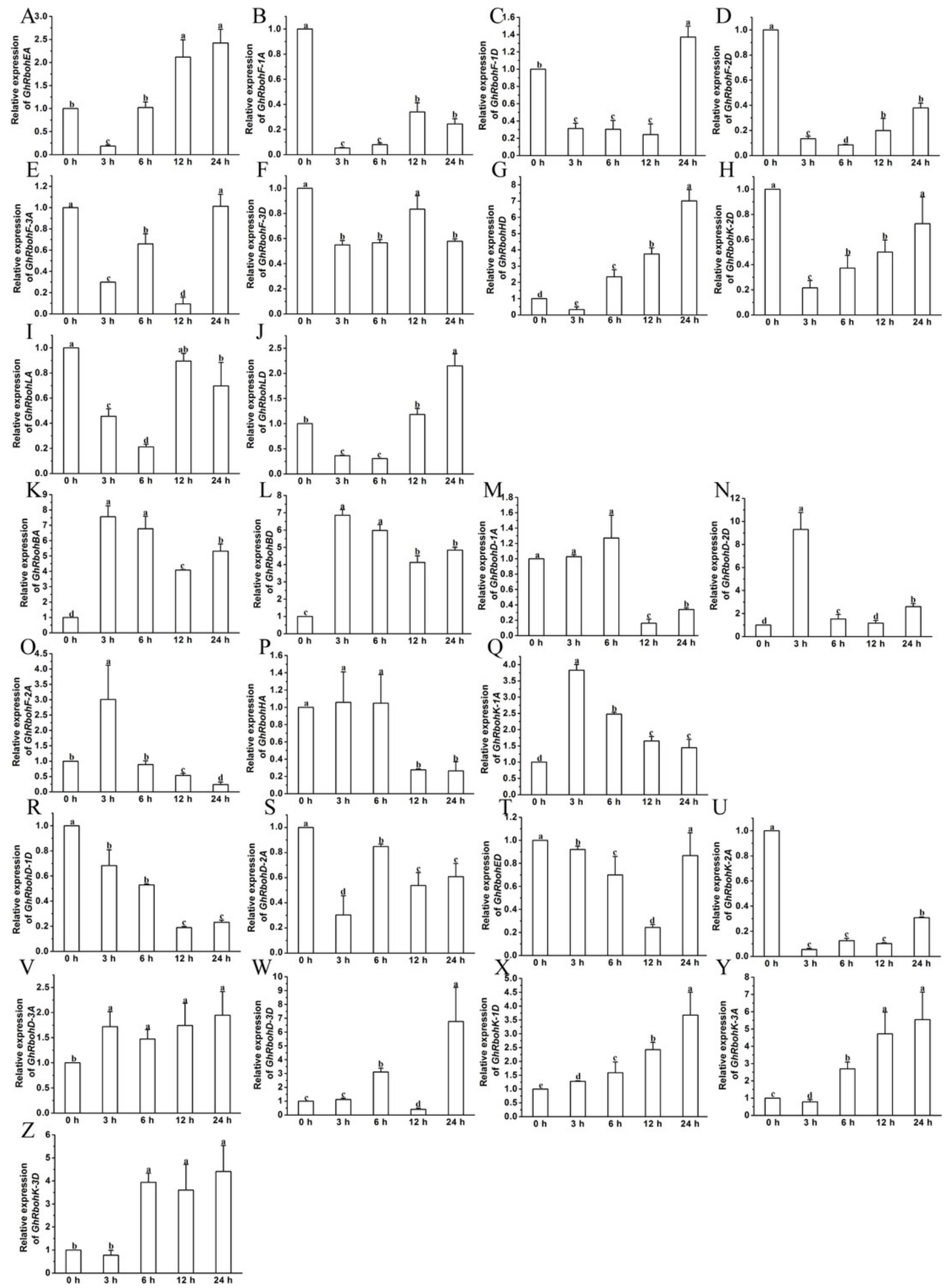

PeerJ reviewing PDF | (2019:08:39951:2:0:NEW 8 Dec 2019) 


\section{Figure 10}

The expression profiles of GhRbohs under high temperature stress.

The expression of the GhRbohs upregulated at $0 \mathrm{~h}, 12 \mathrm{~h}$ and/or $24 \mathrm{~h}$ but downregulated at 3 $\mathrm{h}$ and $6 \mathrm{~h}(\mathrm{~A}-\mathrm{E})$, increased at $3 \mathrm{~h}$ and/or $6 \mathrm{~h}$, but reduced at other time (F-W), continually lowered (X-Y), and elevated (Z). GhUBQ7 acts as the internal control. The relative expression value at $0 \mathrm{~h}$ was set as 1 . Data are presented as mean $\pm \mathrm{SE}$. Different lowercase letters above the error bar show significant differences in gene expression levels at different time points by one-way ANOVA and Tukey's HSD test ( $n \geq 3, P \leq 0.05)$. 

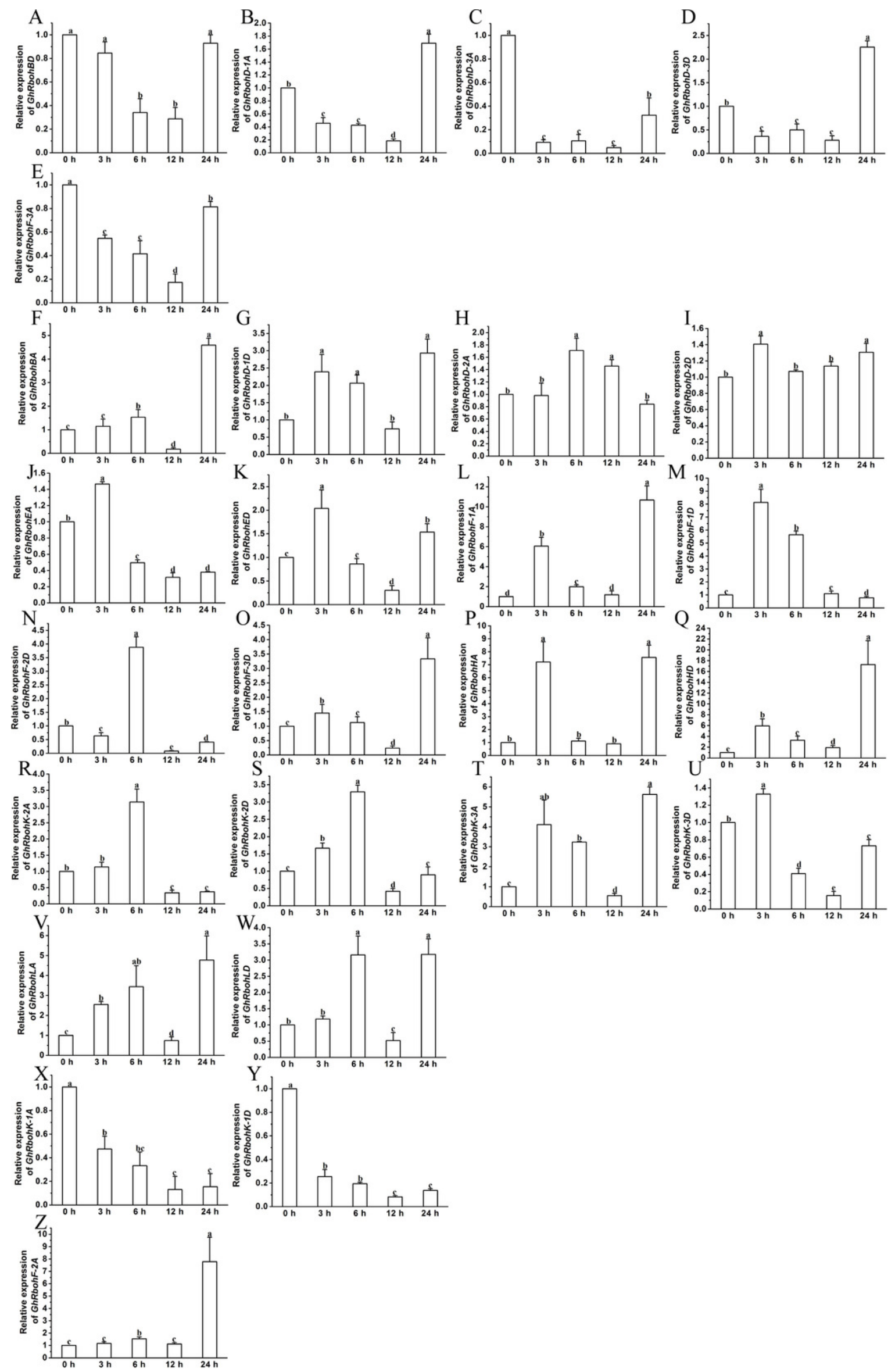

Peer) reviewing PDF | (2019:08:39951:2:0:NEW 8 Dec 2019) 


\section{Figure 11}

Changes in expression of GhRbohs during development of ovules and fibers in cotton.

The heat maps in yellow/white/blue (from low to high) indicate the expression levels of various GhRbohs at different stages of fiber initiation and elongation.

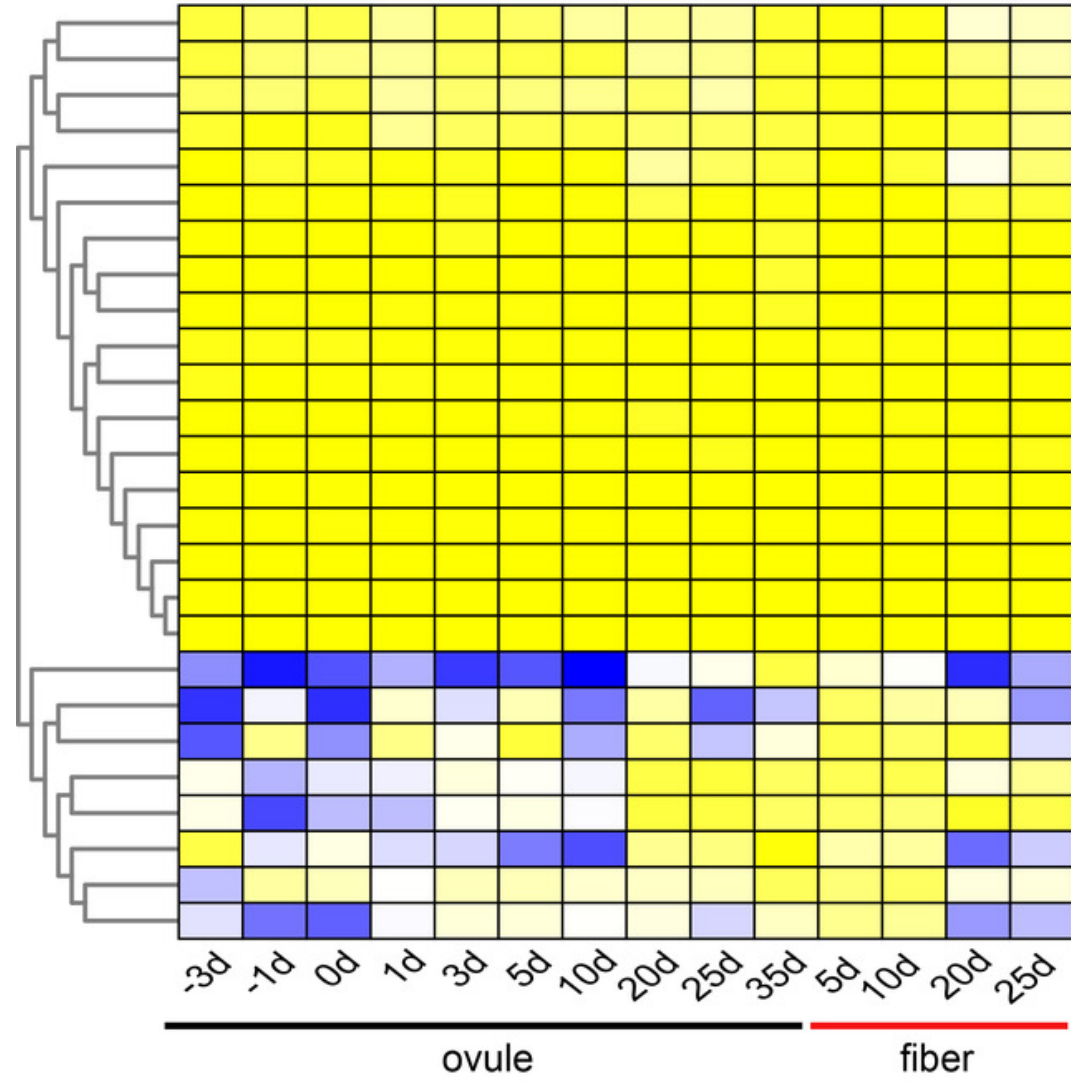

ovule
Gh_A02G1791(GhRbohF-1A)

Gh_A12G2669(GhRbohF-3A)

Gh_D03G0688(GhRbohF-1D)

Gh D12G1807(GhRbohLD)

Gh_A12G2653(GhRbohLA)

Gh_D05G1864(GhRbohD-1D)

Gh_A11G2426(GhRbohBA)

Gh_A03G0476(GhRbohK-3A)

Gh_D07G0136(GhRbohED)

Gh_A08G0982(GhRbohF-2A)

Gh_D08G1257(GhRbohF-2D)

Gh A07G0143(GhRbohEA)

Gh_A05G1666(GhRbohD-1A)

Gh_D01G0990(GhRbohD-2D)

Gh-D11G2743(GhRbohBD)

Gh_A07G0856(GhRbohHA)

Gh_A01G0943(GhRbohD-2A)

Gh_D07G0928(GhRbohHD)

Gh_D12G1932(GhRbohK-2D)

Gh_A05G2211(GhRbohD-3A)

Gh_D05G2471(GhRbohD-3D)

Gh_A07G0398(GhRbohK-1A)

Gh_D07G0463(GhRbohK-1D)

Gh_A12G1774(GhRbohK-2A)

Gh_D03G1062(GhRbohK-3D)

Gh_D12G2750(GhRbohF-3D) 